\title{
SERCA2 Haploinsufficiency in a Mouse Model of Darier Disease Causes a Selective Predisposition to Heart Failure
}

\author{
Vikram Prasad, ${ }^{1}$ John N. Lorenz, ${ }^{2}$ Valerie M. Lasko, ${ }^{2}$ Michelle L. Nieman, ${ }^{2}$ Wei Huang, ${ }^{3}$ \\ Yigang Wang, ${ }^{3}$ David W. Wieczorek, ${ }^{1}$ and Gary E. Shull ${ }^{1}$ \\ ${ }^{1}$ Department of Molecular Genetics, Biochemistry and Microbiology, University of Cincinnati College of Medicine, \\ Cincinnati, OH 45267, USA \\ ${ }^{2}$ Department of Cellular and Molecular Physiology, University of Cincinnati College of Medicine, Cincinnati, OH 45267, USA \\ ${ }^{3}$ Department of Pathology and Laboratory Medicine, University of Cincinnati College of Medicine, Cincinnati, OH 45267, USA
}

Correspondence should be addressed to Vikram Prasad; prasad.vikram@gmail.com

Received 26 September 2014; Revised 18 December 2014; Accepted 23 December 2014

Academic Editor: Monica Fedele

Copyright (C) 2015 Vikram Prasad et al. This is an open access article distributed under the Creative Commons Attribution License, which permits unrestricted use, distribution, and reproduction in any medium, provided the original work is properly cited.

\begin{abstract}
Null mutations in one copy of ATP2A2, the gene encoding sarco/endoplasmic reticulum $\mathrm{Ca}^{2+}$-ATPase isoform 2 (SERCA2), cause Darier disease in humans, a skin condition involving keratinocytes. Cardiac function appears to be unimpaired in Darier disease patients, with no evidence that SERCA2 haploinsufficiency itself causes heart disease. However, SERCA2 deficiency is widely considered a contributing factor in heart failure. We therefore analyzed Atp2a2 heterozygous mice to determine whether SERCA2 haploinsufficiency can exacerbate specific heart disease conditions. Despite reduced SERCA2a levels in heart, Atp2a2 heterozygous mice resembled humans in exhibiting normal cardiac physiology. When subjected to hypothyroidism or crossed with a transgenic model of reduced myofibrillar $\mathrm{Ca}^{2+}$-sensitivity, SERCA2 deficiency caused no enhancement of the disease state. However, when combined with a transgenic model of increased myofibrillar $\mathrm{Ca}^{2+}$-sensitivity, SERCA2 haploinsufficiency caused rapid onset of hypertrophy, decompensation, and death. These effects were associated with reduced expression of the antiapoptotic Haxl, increased levels of the proapoptotic genes Chop and Casp12, and evidence of perturbations in energy metabolism. These data reveal myofibrillar $\mathrm{Ca}^{2+}$-sensitivity to be an important determinant of the cardiac effects of SERCA2 haploinsufficiency and raise the possibility that Darier disease patients are more susceptible to heart failure under certain conditions.
\end{abstract}

\section{Introduction}

In humans, loss of one copy of the ATP2A2 gene causes Darier disease (DD), an acantholytic skin disease $[1,2]$. ATP2A2 encodes two alternatively spliced variants of sarco/endoplasmic reticulum $\mathrm{Ca}^{2+}$-ATPase isoform 2 (SERCA2). These are SERCA2b, the ubiquitous endoplasmic reticulum (ER) $\mathrm{Ca}^{2+}$ pump, and SERCA2a, the cardiac and slow-twitch skeletal muscle sarcoplasmic reticulum (SR) $\mathrm{Ca}^{2+}$ pump [3]. In its capacity as the primary cardiac SR Ca ${ }^{2+}$ pump, SERCA2a facilitates muscle relaxation and replenishes $\mathrm{SR} \mathrm{Ca}^{2+}$ stores needed for contraction [3]. Because reduced SERCA2 activity is often observed in heart disease [4] and DD mutations cause reductions in SERCA2 expression and activity [5], one might expect these mutations to lead to heart disease in humans. However, two studies of DD patients with a mean age of $\sim 47$ years indicated that cardiac performance is normal and yielded no evidence of heart disease [6,7]; these findings have been interpreted to suggest that a role for SERCA2 deficiency in heart disease, while relevant in rodent models, may be less important in humans [7]. However, while these results provide compelling evidence that Atp $2 a 2$ heterozygosity does not impair cardiac function in middle-aged humans, they do not rule out the possibility that SERCA2 haploinsufficiency can increase susceptibility to disease progression and heart failure.

In earlier studies, ablation of one copy of the Atp2a2 gene in mice of a mixed 129Svj and Black Swiss background caused 
a reduction in cardiac SERCA2a protein expression to $65 \%$ of wild-type (WT) levels, with impaired contractility and relaxation [8]. However, true heart disease was not observed; in fact, the major phenotype of the Atp $2 a 2^{+/-}$mice was the development of squamous cell tumors in keratinized epithelial tissues $[9,10]$. Later studies on these mice revealed an increased susceptibility to pressure-overload cardiac hypertrophy [11] and a reduction of rate-dependent inotropy in isolated mutant hearts, relative to $\mathrm{WT}$ controls [12]. In the current study, we used Atp $2 a 2^{+/-}$mice on an inbred FVB/N background; while these heterozygous (HET) mice continued to display effects of SERCA2 haploinsufficiency in keratinized epithelia, cardiac performance was apparently normal, more closely reflecting findings in DD patients $[6,7]$. The HET model was well suited to determine if SERCA2 deficiency, while apparently benign under normal conditions, could exacerbate cardiac disease progression. Specifically, we investigated the interaction of SERCA2 haploinsufficiency with hypothyroidism, which is known to impair cardiac performance [13], and alterations in myofibrillar $\mathrm{Ca}^{2+}$-sensitivity, which causes pathological hypertrophy and heart failure $[14,15]$. For the latter, double mutant mice were generated by crossing HET mice with transgenic lines expressing the Glu54Lys mutant $\alpha$-tropomyosin, which reduces myofibrillar $\mathrm{Ca}^{2+}$-sensitivity and leads to dilated cardiomyopathy $[16,17]$, and the Glu180Gly mutant $\alpha$-tropomyosin, which increases myofibrillar $\mathrm{Ca}^{2+}$ sensitivity and causes hypertrophic cardiomyopathy $[18,19]$. Our results reveal an unexpected selectivity in the effects of SERCA2 haploinsufficiency in heart, which should be taken into consideration in the management of Darier disease patients.

\section{Materials and Methods}

2.1. Animal Models. The original Atp2a $22^{+/-}$line was backcrossed onto the inbred FVB/N background in excess of 15 generations to generate the HET mutant line utilized in this study. This Atp $2 a 2^{+/-}$mouse line has been made available to researchers through the Jackson Labs repository. All other mice used in this study, including the WT, the transgenic mouse models carrying the Glu154Lys mutant $\alpha$-tropomyosin, which has dilated cardiomyopathy (DCM) [16], and the Glu180Gly mutant $\alpha$-tropomyosin, which has hypertrophic cardiomyopathy (HCM) [18], were also on the inbred FVB/N background. HET mice were bred with the DCM and HCM lines to generate double (DCM/HET and HCM/HET) and single mutant offspring. The hypothyroid model was prepared by treating WT and HET mice with 6-n-propyl thiouracil (PTU) exactly as previously described [20]. All procedures conformed to guidelines published by the NIH (Guide for the Care and Use of Laboratory Animals; publication number 86-23, revised 1996) and were approved by the Institutional Animal Care and Use Committee at the University of Cincinnati.

2.2. Evaluation of Cardiac Function. Anesthesia of mice with ketamine and inactin, analysis of cardiovascular function using pressure transducers inserted into the left ventricle and right femoral vein, delivery of drugs via a cannula in the right femoral vein, and recording and analysis of data were performed exactly as described previously [13]. Analysis of cardiac function by M-mode echocardiography of mice, anesthetized using isoflurane inhalation, and analysis of data were performed exactly as described previously [21].

2.3. Immunoblot Analyses. Hearts were harvested from anesthetized mice and processed for immunoblot analysis as previously described [22]. Phosphorylation of phospholamban (PLN) in response to $\beta$-adrenergic stimulation was assessed in ventricles from mice that were anesthetized and surgically instrumented as described above and treated with dobutamine (16 ng/g body weight/min). Estimation of protein concentration in total homogenates, resolution of proteins by discontinuous, reducing SDS-PAGE, and immunoblot analyses were carried out as described [22]. All primary and secondary antibodies used have been previously described [22].

2.4. Real-Time Polymerase Chain Reaction. Hearts/ventricles were harvested from anesthetized mice and processed for real-time PCR (RT-PCR) analysis as previously described [22]. In addition to the primer pairs that have been previously described [22, 23], the following were used: Hspa5 (GRP78/BiP), PrimerBank ID number 31981722al; Hsp90b1 (GRP94), PrimerBank ID number 6755863al; Casp12 (Caspase 12), PrimerBank ID number 31981868a1; Ddit3 (CHOP), PrimerBank ID number 31982415a1; Eif2ak3 (PERK), PrimerBank ID number 6857781al; Acoxl, PrimerBank ID number 26333821al; Fabp3, PrimerBank ID number 6753810a1; Orail, PrimerBank ID number 93277106b1; Stim1, PrimerBank ID number 31981983a2; Hax1, PrimerBank ID number 6754160al; Rcan2, PrimerBank ID number 46560586cl; and Ppar $\gamma$, PrimerBank ID number 187960104cl; primers for $\mathrm{Pln}$ (phospholamban) were adapted from PrimerBank ID number 213512815cl (forward primer: 5' AAGTGCAATACCTCACTCG 3', reverse primer: 5' GATCAGCAGCAGACATATC $3^{\prime}$ ). mRNA levels for Atp2b1 (QT01072106) and Atp2b4 (QT01076271) were determined using QuantiTect Primer Assay Kits (Qiagen).

2.5. Statistics. Results are presented as means \pm standard error (SE). Individual comparisons were performed using a two-sided Student's $t$-test, and a $P$ value of $<0.05$ was considered significant.

\section{Results}

3.1. Cardiovascular Performance in WT and HET Mice. Cardiovascular performance of adult FVB/N WT and HET mice was analyzed using a pressure transducer in the left ventricle under both basal conditions and upon $\beta$-adrenergic stimulation with dobutamine. No significant differences were observed in basal heart rate, mean arterial pressure, left ventricular end-diastolic pressure, or maximum rates of left ventricular pressure development or decay (Figure 1). Treatment with dobutamine led to similar changes in both genotypes, with no impairment of chronotropic, inotropic, or lusitropic responses. Rate of left ventricular pressure development was 


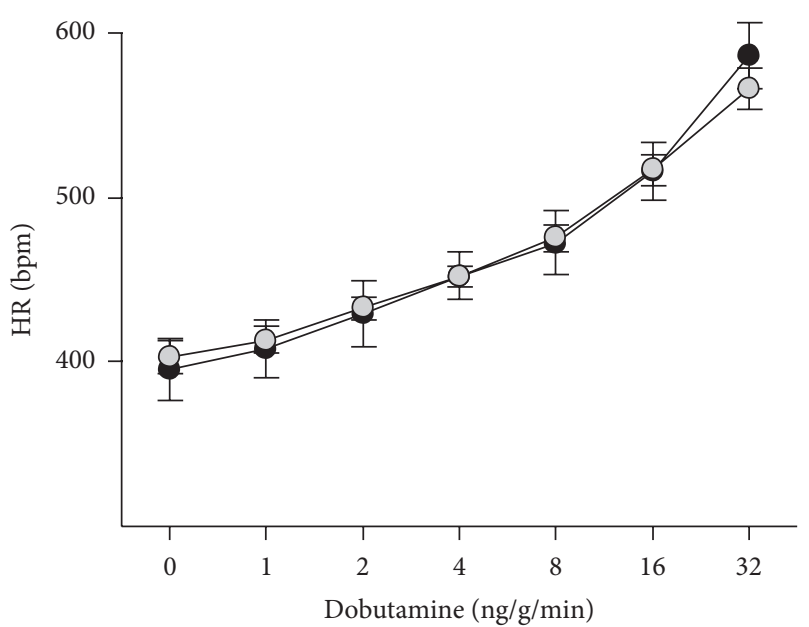

(a)

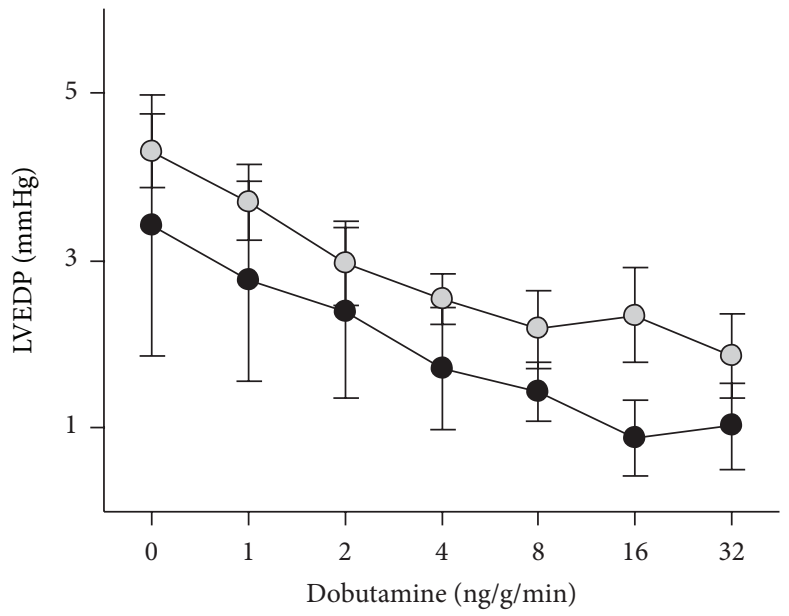

(c)

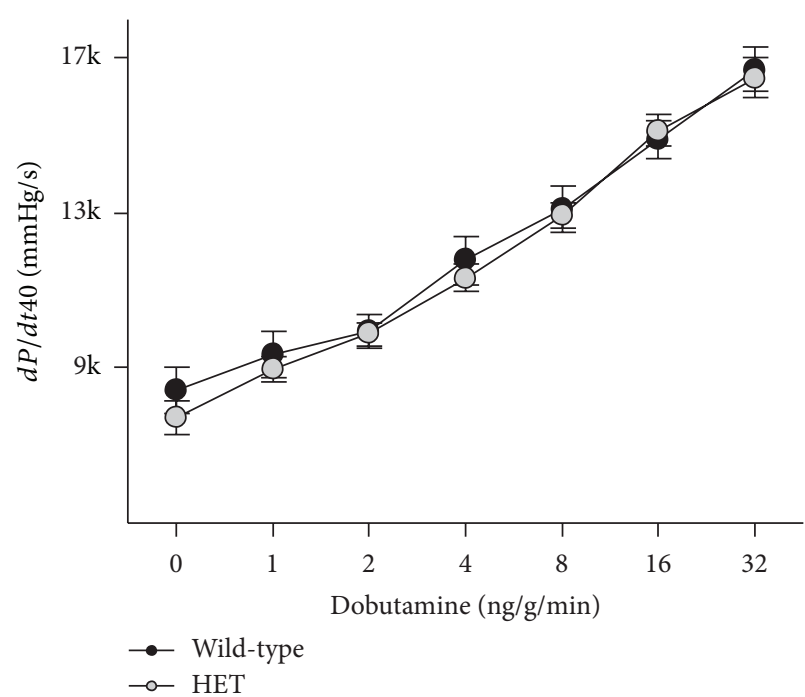

(e)

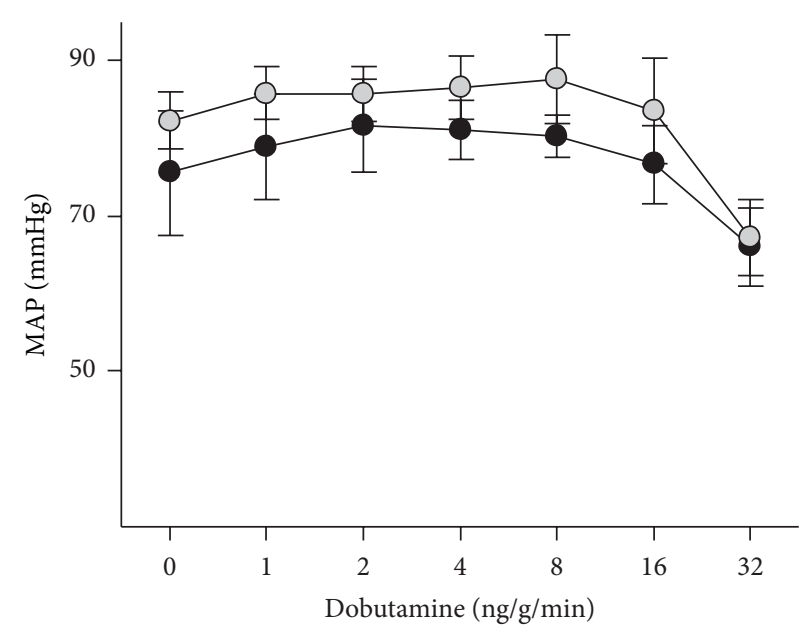

(b)

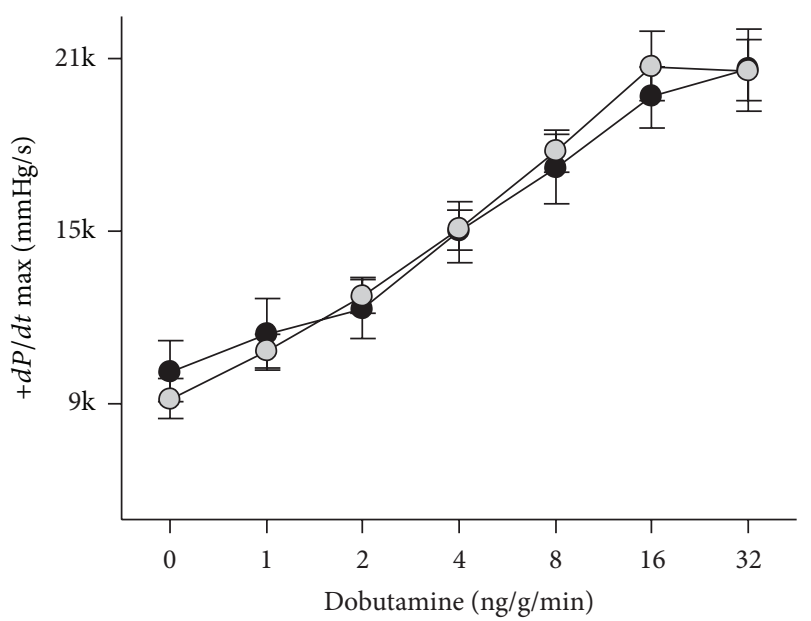

(d)

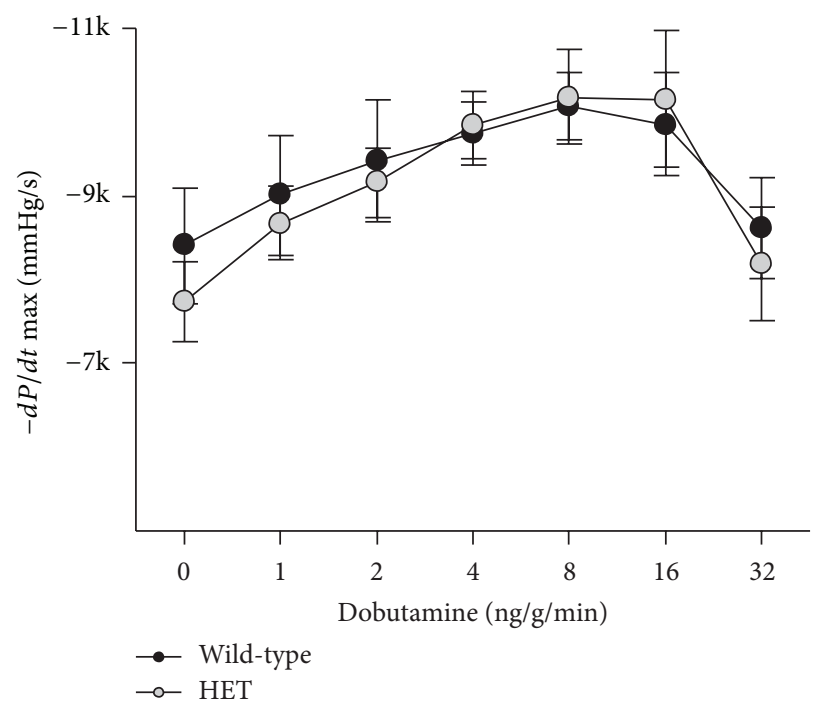

(f)

FIgure 1: Cardiovascular function in Atp2a2 heterozygous mice. Ventricular and arterial pressures were measured in anesthetized adult FVB/N wild-type and Atp2a2 $2^{+/-}$mice under baseline conditions and upon $\beta$-adrenergic stimulation using dobutamine. (a) Heart rate (HR); (b) mean arterial pressure (MAP); (c) left ventricular end-diastolic pressure (LVEDP); (d) maximal rate of left ventricular pressure development (+dP/dt Max); (e) rate of left ventricular pressure development at $40 \mathrm{mmHg}(\mathrm{dP} / \mathrm{dt} 40)$; and (f) maximal rate of decay of left ventricular developed pressure (-dP/dt Max). Values are means \pm SE. $n=$ at least 5 for each genotype. 


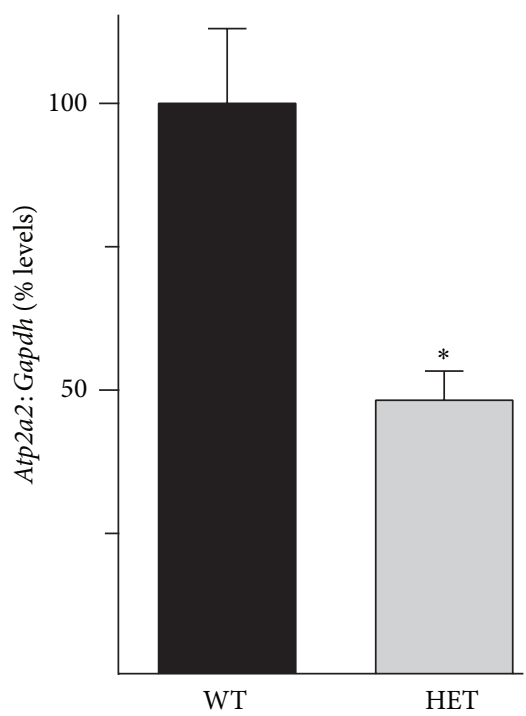

(a)

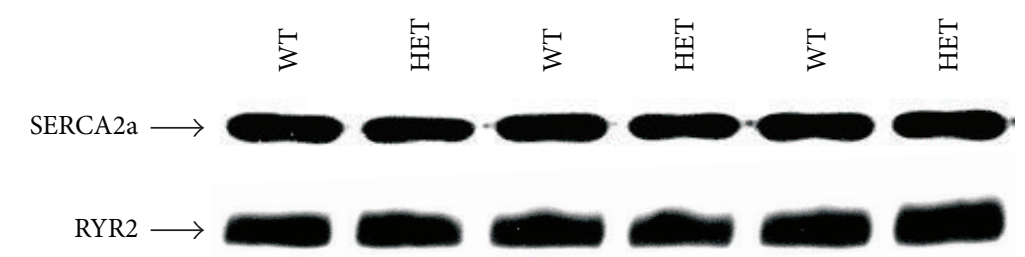

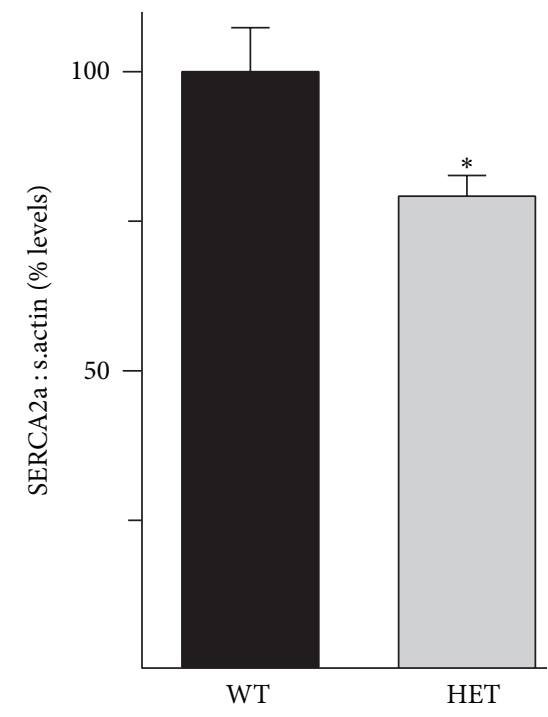

(c)

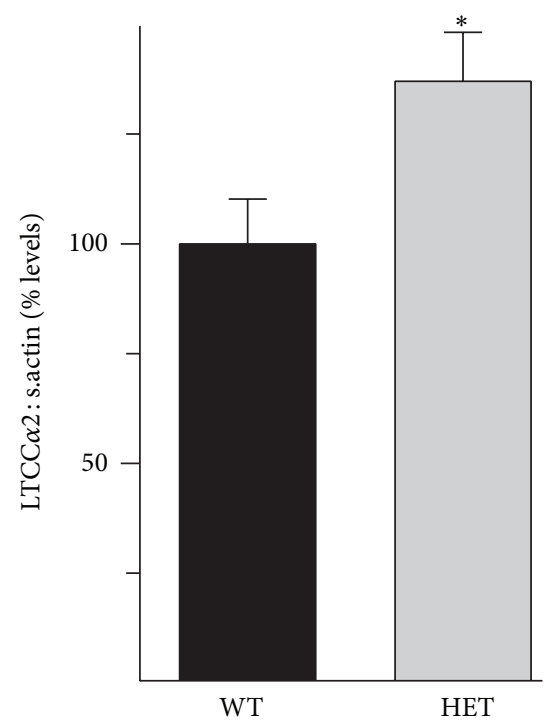

(d)

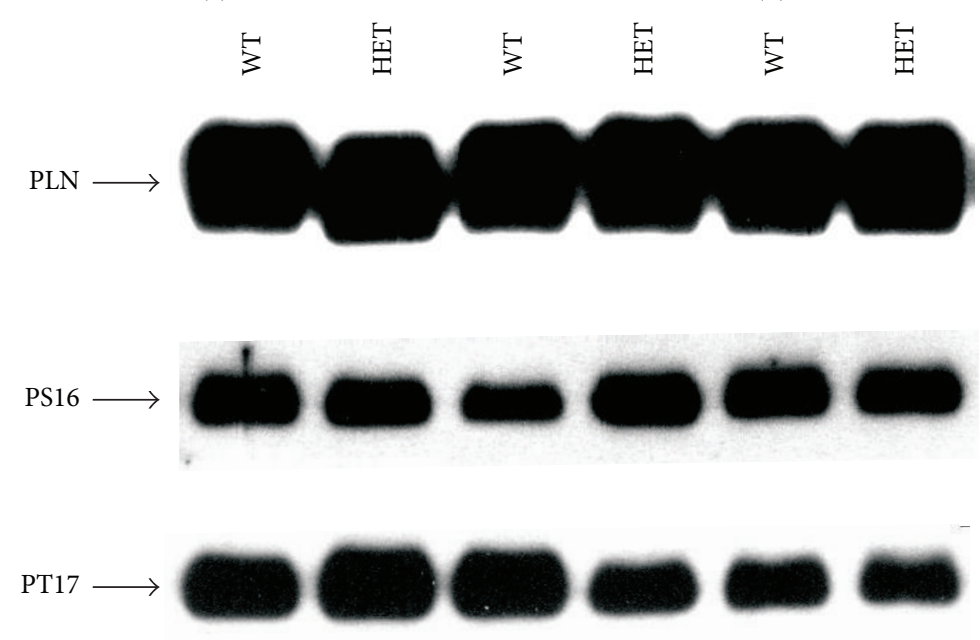

(e)

Figure 2: Continued. 


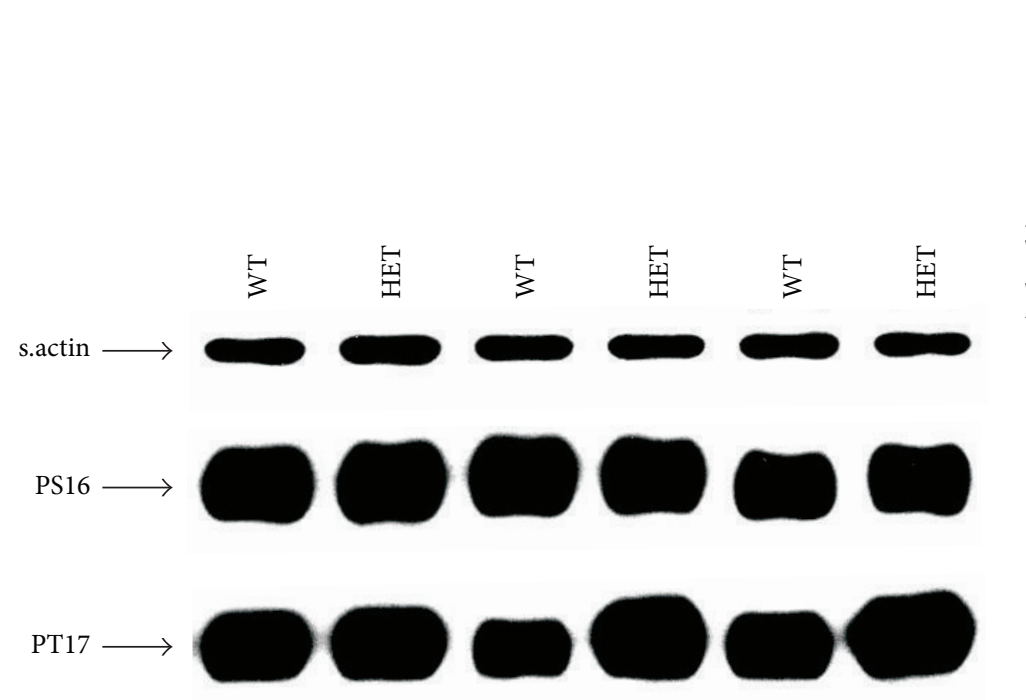

(f)

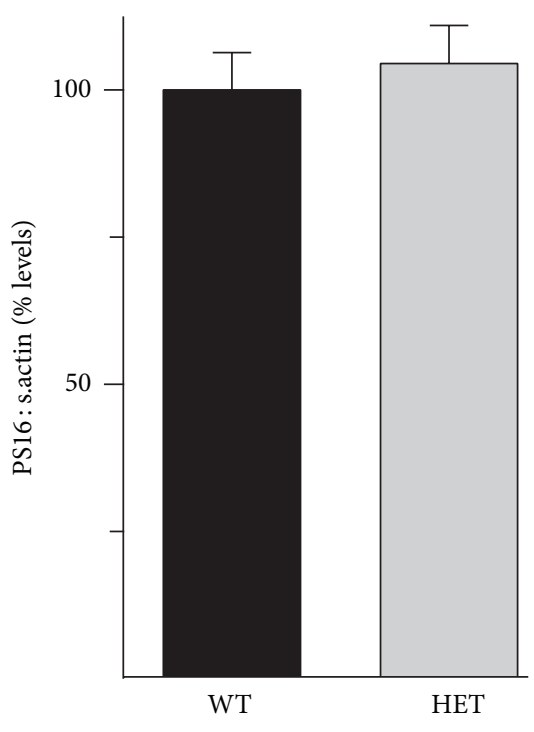

(g)

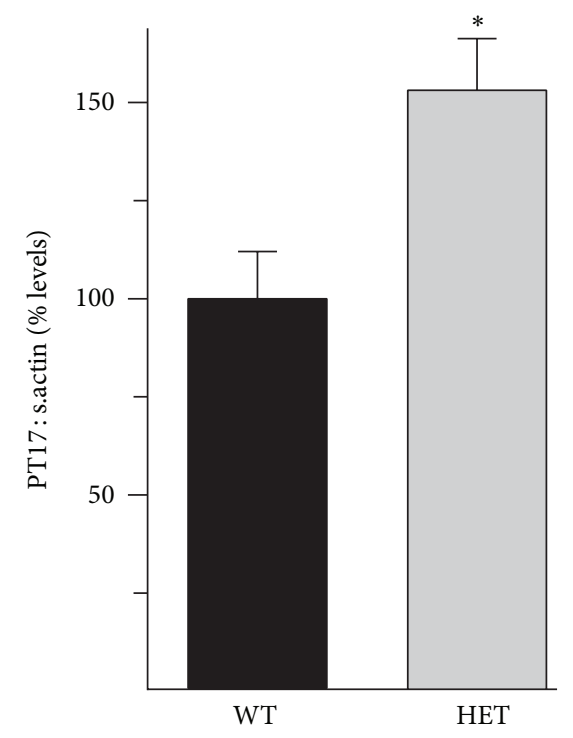

(h)

FIGURE 2: Effects of Atp2a2 heterozygosity on expression of $\mathrm{Ca}^{2+}$ handling proteins in heart. Adult WT and Atp2a2 ${ }^{+/-}$(HET) hearts were processed for analysis of mRNA and protein levels. (a) Atp2a2 mRNA determined by RT-PCR; (b) immunoblot analysis of SERCA2a, ryanodine receptor isoform 2 (RYR2), $\alpha 2$ subunit of L-type $\mathrm{Ca}^{2+}$ channel (LTCC $\alpha 2$ ), and $\mathrm{Na}^{+} / \mathrm{Ca}^{2+}$ exchanger isoform 1 (NCX1). Quantitation of SERCA2a (c) and LTCC $\alpha 2$ (d) protein levels. Immunoblot analyses of phospholamban (PLN) and PLN phosphorylated on Ser16 (PS16) and Thr17 (PT17) were performed using heart samples from anesthetized surgically instrumented mice under both baseline conditions (e) and after $\beta$-adrenergic stimulation with dobutamine at $16 \mathrm{ng} / \mathrm{g}$ body weight $/ \mathrm{min}$ (f, g, h). mRNA levels were normalized to Gapdh and protein levels were normalized to sarcomeric actin (s.actin). Values are means \pm SE. $n=$ at least 4 for each genotype. ${ }^{*} P<0.05$ versus WT controls.

also calculated at $40 \mathrm{~mm} \mathrm{Hg}$, to assess possible effects of the small but nonsignificant difference in mean arterial pressure (Figure 1(b)); however the two genotypes had virtually identical responses.

\subsection{Effects of Atp2a2 Heterozygosity on Proteins Implicated in} $\mathrm{Ca}^{2+}$-Handling. RT-PCR analysis of total RNA from WT and HET hearts revealed that mRNA levels of the Atp2a2 gene, which codes for SERCA2, were reduced to $48 \pm 5 \%$ of WT levels (Figure 2(a)). However, immunoblot analysis of total protein homogenates revealed a much smaller reduction in SERCA2a protein levels (to $79 \pm 3 \%$ of WT levels, Figures 2(b) and $2(\mathrm{c}))$ in HET hearts. This reduction was associated with an increase (to $137 \pm 11 \%$ of WT levels, Figures 2(b) and 2(d)) in expression of the $\alpha 2$ subunit of the L-type $\mathrm{Ca}^{2+}$-channel, which is the principal $\mathrm{Ca}^{2+}$-uptake mechanism on a beat-tobeat basis. There were no changes in expression of either the ryanodine receptor isoform 2 , which mediates $\mathrm{Ca}^{2+}$-release 
from the SR, or the $\mathrm{Na}^{+} / \mathrm{Ca}^{2+}$ exchanger isoform 1 (NCXl, Slc8a1), which is the predominant $\mathrm{Ca}^{2+}$-efflux mechanism in cardiac myocytes, in HET hearts (Figure 2(b)).

SERCA2 activity is negatively regulated by phospholamban (PLN), which binds to the $\mathrm{Ca}^{2+}$-pump in a phosphorylation-dependent manner [24]. Reduced PLN expression and increased PLN phosphorylation on residues Ser16 (PS16) and Thr17 (PT17) can both enhance SERCA2a-mediated $\mathrm{SR} \mathrm{Ca}^{2+}$-sequestration [24]. However, immunoblot analysis revealed that PLN expression and baseline phosphorylation (Figure 2(e)) were unaltered in HET hearts. The inotropic and lusitropic effects of $\beta$-adrenergic stimulation are mediated, at least in part, via increased PLN phosphorylation, which facilitates more robust $\mathrm{Ca}^{2+}$-cycling [24]. The normal increase in the cardiovascular performance of stimulated HET mice raised the possibility that $\beta$-adrenergic stimulated PLN phosphorylation was augmented to compensate for the reduction in SERCA2a levels. To test this hypothesis, PS16 and PT17 levels were determined in hearts from WT and HET mice stimulated with dobutamine. While PS16 levels were comparable between stimulated WT and HET hearts (Figures 2(f) and 2(g)), PT17 levels were elevated in stimulated HET hearts (by $153 \pm 13 \%$ ), when compared to similarly treated WT hearts (Figures 2(f) and 2(h)).

\subsection{Atp2a2 Heterozygosity Does Not Exacerbate Cardiac} Dysfunction Caused by Hypothyroidism. Hypothyroidism is a well-recognized cardiovascular disease risk factor [25]. Studies in rabbits and mice have shown that it is associated with a reduction in cardiac SERCA2 expression [20, 26], raising the possibility that the impairment of cardiac function caused by hypothyroidism would be greater in Atp2a2 heterozygous individuals. To test this hypothesis, hypothyroidism was induced in WT and HET mice and cardiac performance was assessed by in vivo catheterization. Cardiovascular function was similarly diminished in both WT and HET mice (Figures 3(a)-3(f)) under both baseline conditions and upon $\beta$-adrenergic stimulation.

3.4. Atp2a2 Heterozygosity Has No Appreciable Effect on the Cardiac Phenotype of Transgenic Mice with Dilated Cardiomyopathy. Myofibrillar $\mathrm{Ca}^{2+}$-sensitivity is a major determinant of cardiac function; in addition to altering force development, changes in $\mathrm{Ca}^{2+}$-sensitivity can impact myofibrillar $\mathrm{Ca}^{2+}$-buffering, diastolic $\mathrm{Ca}^{2+}$-levels, and SERCA2-mediated cytosolic $\mathrm{Ca}^{2+}$-clearance $[14,15,27-29]$. Transgenic expression of Glu154Lys mutant $\alpha$-tropomyosin in the DCM mouse heart lowers myofibrillar $\mathrm{Ca}^{2+}$ sensitivity, impairs contractility, and leads to dilated cardiomyopathy [16]. In order to determine the effects of SERCA2 haploinsufficiency, double mutant DCM/HET mice were analyzed. DCM/HET mice were viable and appeared normal. The increase in heart weight: body weight (HW:BW) and heart weight:tibial length ratios, determined in 8-10-week-old mice, was similar in both single mutant DCM and double mutant DCM/HET mice (Figure 4(a)). Echocardiographic analyses revealed that cardiac function was not compromised in DCM/HET mice when compared to DCM controls; in fact, fractional shortening and ejection fraction trended slightly higher in DCM/HET hearts (Figure 4(b)). RT-PCR analysis showed that the increases in mRNA levels for $N p p a$ (atrial natriuretic peptide), Myh7 ( $\beta$-myosin heavy chain), and Actal (skeletal $\alpha$-actin), as markers of pathological hypertrophy, were not significantly different between DCM and DCM/HET hearts (Figure 4(c)). Atp2a2 mRNA levels, which were reduced to $69 \pm 4 \%$ of WT levels in DCM hearts, were lower in DCM/ HET hearts ( $42 \pm 4 \%$ of WT levels; Figure 4(d)). However, the reduction in SERCA2a protein was more modest, with expression at $86 \pm 2 \%$ of DCM levels in DCM/HET hearts (Figure 4(e)).

3.5. Atp2a2 Heterozygosity Causes Rapid Progression of Hypertrophy and Decompensation in Transgenic HCM Mice. Increased myofibrillar $\mathrm{Ca}^{2+}$-sensitivity, which impairs relaxation, is associated with hypertrophic cardiomyopathy and has also been reported in end-stage heart failure [14, 15]. Cardiac expression of Glu180Gly mutant $\alpha$-tropomyosin in the HCM transgenic model increases myofibrillar $\mathrm{Ca}^{2+}$ sensitivity, impairs relaxation, leads to development of fibrosis and hypertrophy, and causes death at 5-6 months of age [18]. To determine the effects of SERCA2 haploinsufficiency on the HCM phenotype, double mutant HCM/HET mice were generated as described above. SERCA2 haploinsufficiency caused a rapid onset of heart failure and death; $80 \%$ of HCM/HET mice were dead by 5 weeks of age and none survived beyond 6-7 weeks (Figure 5(a)). When compared to age-matched HCM controls, HCM/HET hearts displayed overt hypertrophy with pronounced left atrial remodeling as early as 4 weeks of age (Figure 5(b)). HW : BW ratios were higher in HCM/HET mice (Figure 5(c)), as were ratios of ventricular weight : body weight (Figure 5(d)), indicating that the increase in HW : BW was not simply a consequence of atrial remodeling. Consistent with these changes, mRNA levels for several markers of cardiac remodeling, which have been shown to be elevated in 4-week-old HCM hearts [23], were further increased in HCM/HET hearts; these included Nppa (Figure 5(e)), Myh7 (Figure 5(f)), Acta1 (Figure 5(g)), and Ctgf, encoding connective tissue growth factor (Figure $5(\mathrm{~h}))$.

3.6. Effect of Atp2a2 Heterozygosity on Regulators of $\mathrm{Ca}^{2+}$ Handling and Protein Phosphatases in HCM/HET Hearts. RT-PCR analysis of 4-week-old WT, HCM, and HCM/HET hearts revealed that Atp2a2 mRNA levels, which were reduced to $72 \pm 5 \%$ of WT levels in HCM hearts, were sharply reduced in HCM/HET hearts (to $18 \pm 2 \%$ of WT levels; Figure 6(a)). mRNA levels for PLN, which were maintained at WT levels in HCM hearts, were also reduced in HCM/HET hearts ( $53 \pm 3 \%$ of WT levels; Figure 6(b)). However, expression of SERCA2a protein showed a smaller reduction of just $23 \pm 5 \%$ from HCM levels in HCM/HET hearts (Figures 6(c) and 6(d)), with no change seen in PLN protein levels (Figure 6(c)).

In addition to its role in contractility, $\mathrm{Ca}^{2+}$ is a regulator of key signaling cascades in heart; the $\mathrm{Ca}^{2+}$-pools 


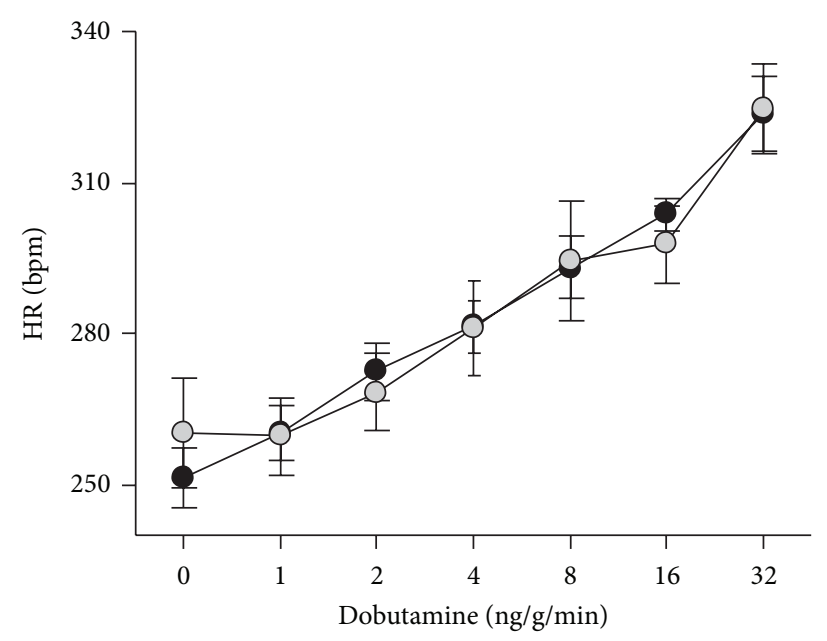

(a)

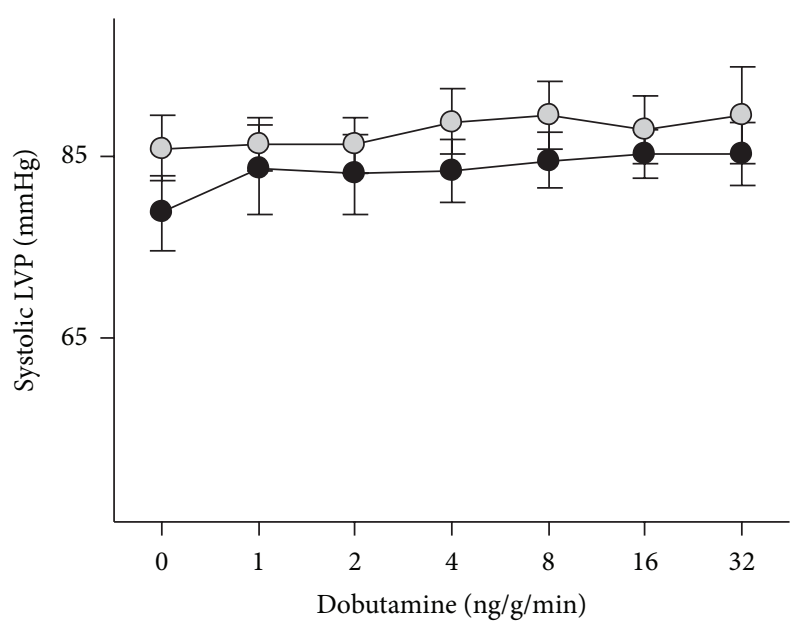

(c)

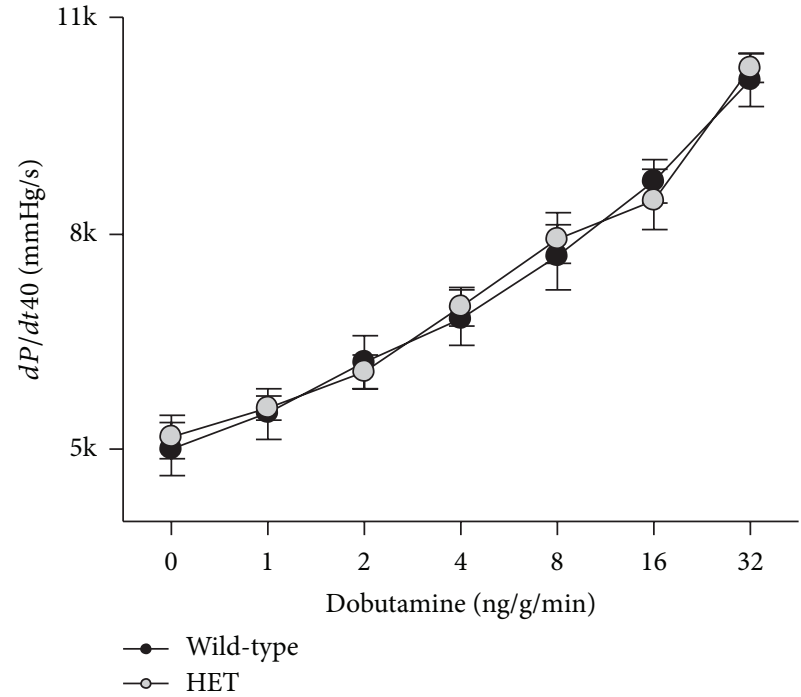

(e)

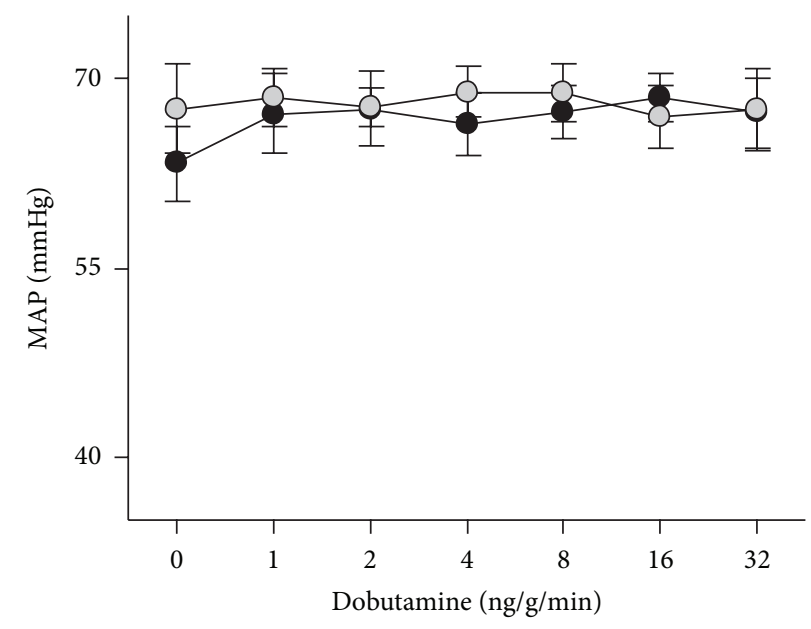

(b)

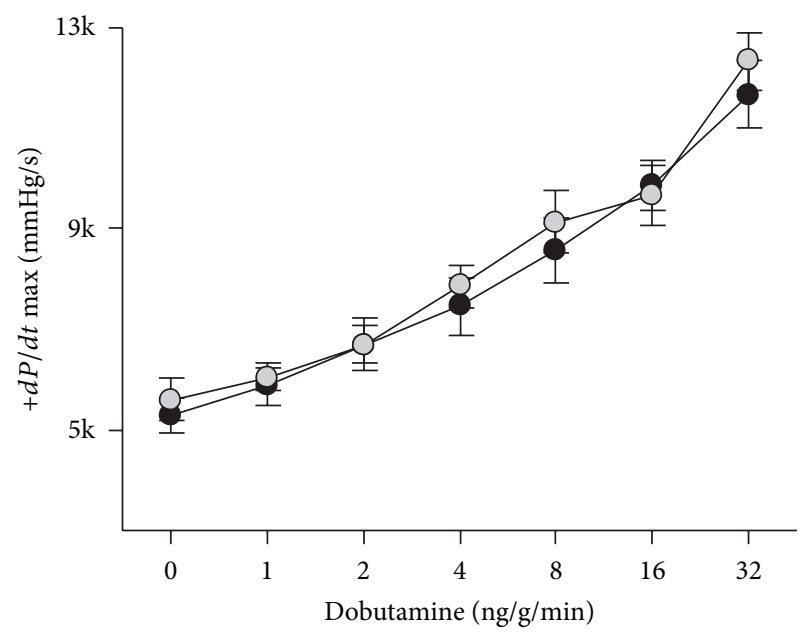

(d)

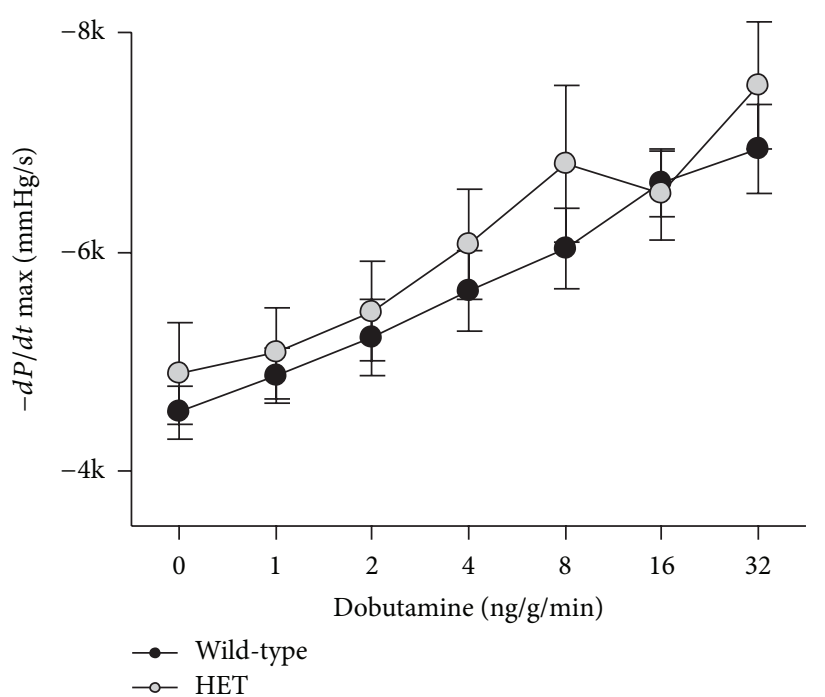

(f)

FIGURE 3: Effects of hypothyroidism on cardiovascular performance of Atp2a2 heterozygous mice. Adult wild-type and Atp2a $2^{+/-}$(HET) mice were rendered hypothyroid and cardiovascular performance was assessed under both baseline conditions and after $\beta$-adrenergic stimulation. (a) Heart rate (HR); (b) mean arterial pressure (MAP); (c) systolic left ventricular pressure (systolic LVP); (d) maximal rate of left ventricular pressure development $(+\mathrm{dP} / \mathrm{dt} \mathrm{Max})$; (e) rate of left ventricular pressure development at $40 \mathrm{mmHg}(\mathrm{dP} / \mathrm{dt} 40)$; (f) maximal rate of decay of left ventricular developed pressure (-dP/dt Max). Values are means \pm SE. $n=$ at least 4 for each genotype. 

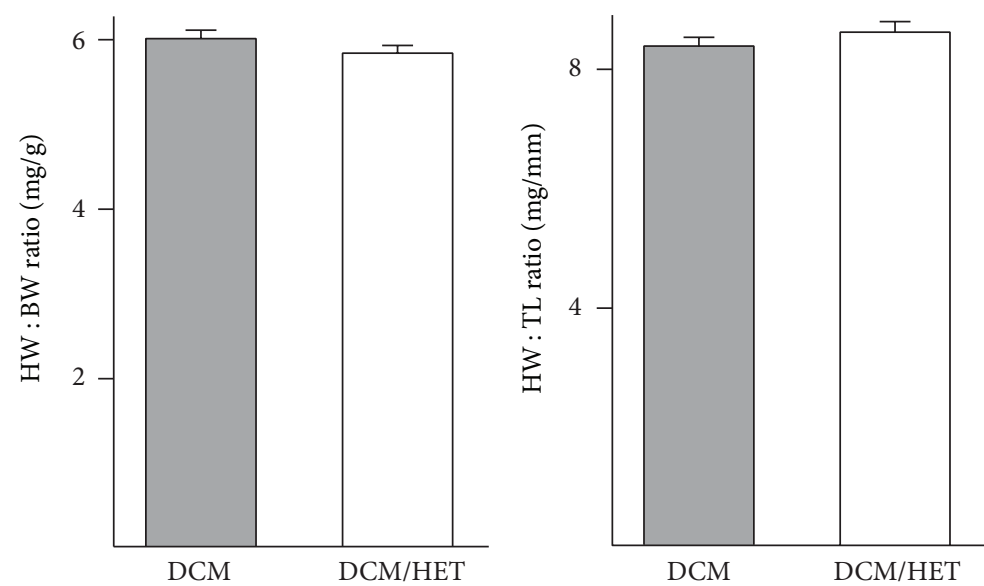

(a)
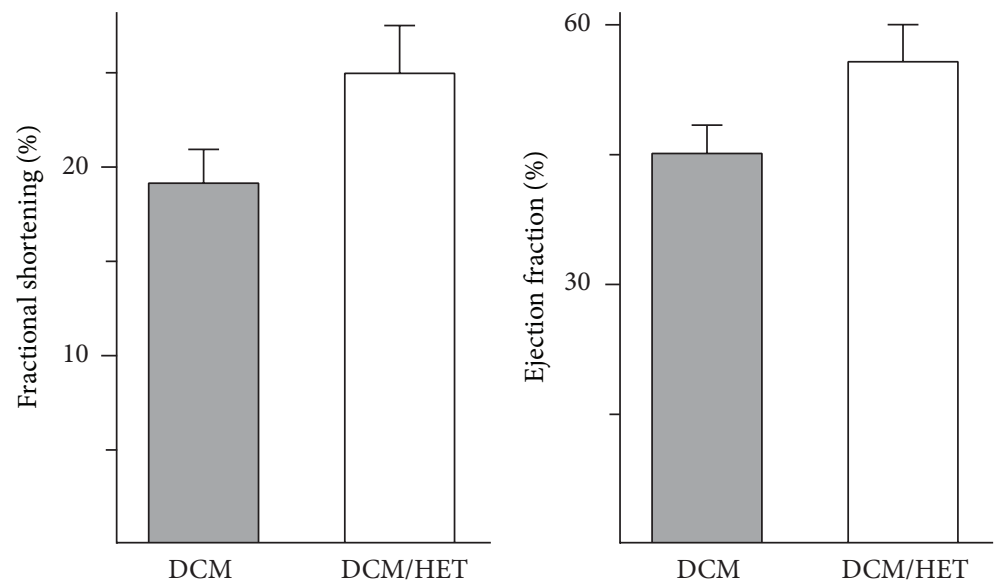

(b)
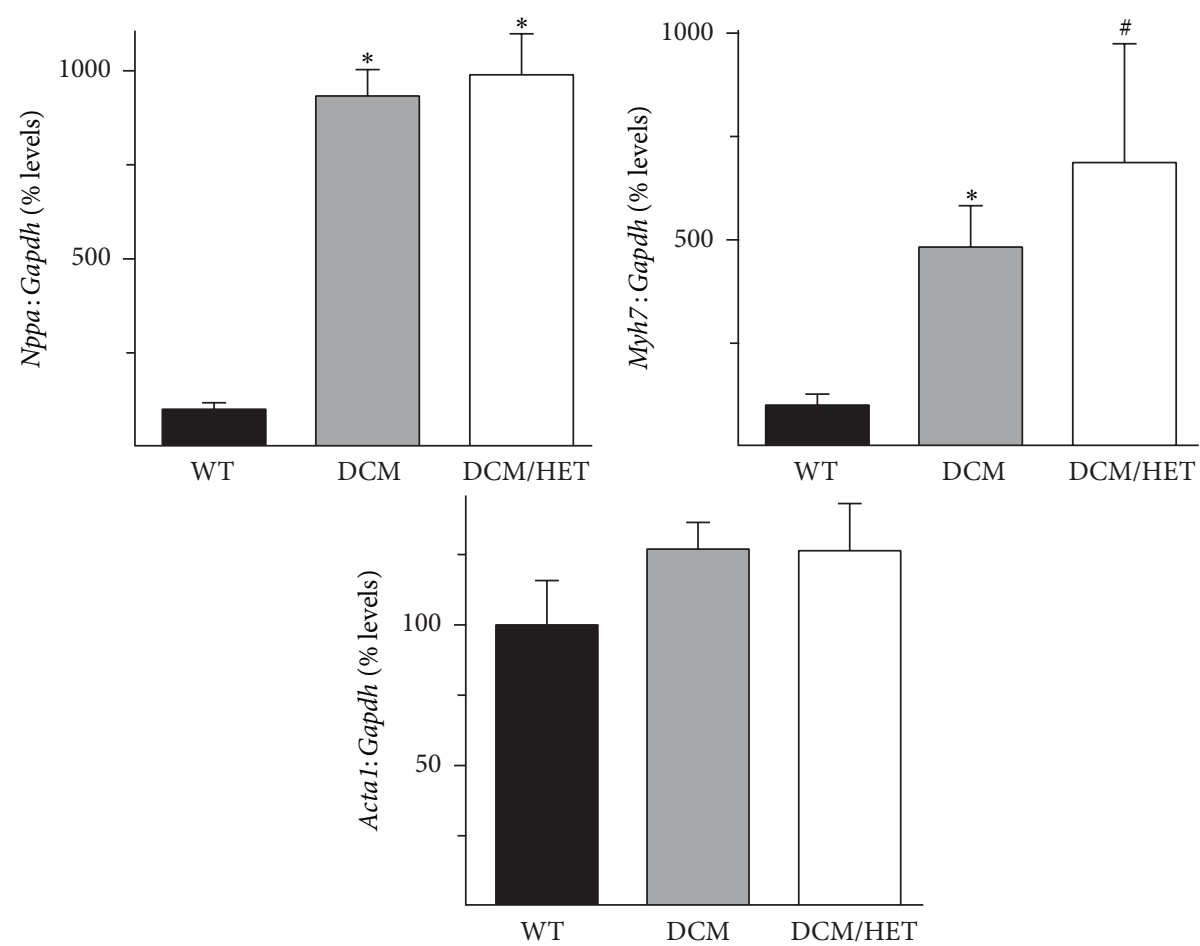

(c)

Figure 4: Continued. 


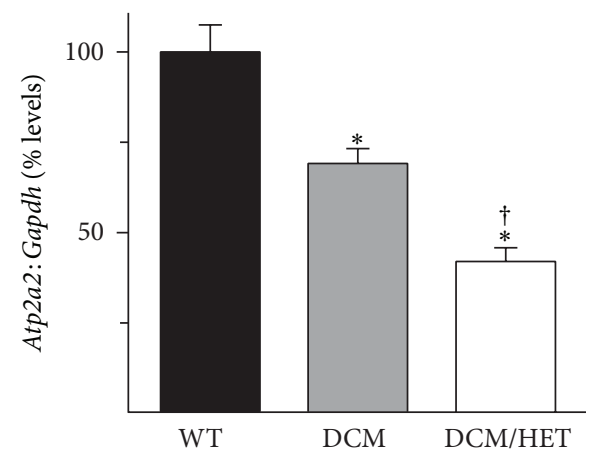

(d)

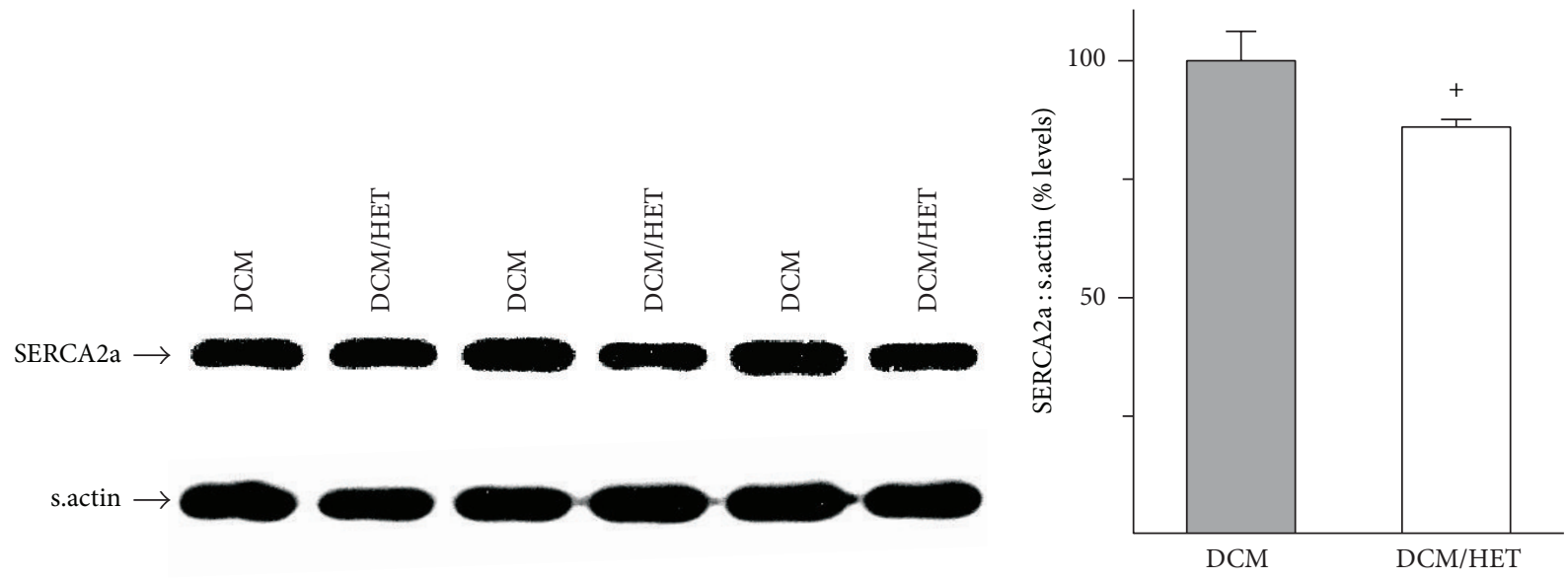

(e)

FIGURE 4: Effects of Atp2a2 heterozygosity in a transgenic model of reduced myofibrillar $\mathrm{Ca}^{2+}$ sensitivity. WT mice, transgenic mice expressing the Glu154Lys mutant $\alpha$-tropomyosin, which causes dilated cardiomyopathy (DCM), and double mutant DCM/Atp2a2 $2^{+-}$(DCM/HET) mice were analyzed. Morphometric analyses revealed similar heart weight : body weight (HW : BW) and heart weight : tibial length (HW : TL) ratios in DCM and DCM/HET mice (a); echocardiographic analysis shows fractional shortening and ejection fraction in DCM and DCM/HET mice (b); RT-PCR analysis shows mRNA levels for (c) atrial natriuretic peptide (Nppa), $\beta$-myosin heavy chain $(M y h 7)$, and skeletal $\alpha$-actin (Acta1) and for Atp2a2 (d). Immunoblot analysis of cardiac homogenates and quantitation show relative levels of SERCA2a (e) in DCM and DCM/HET hearts. mRNA levels were normalized to Gapdh and protein levels were normalized to sarcomeric actin (s.actin). Values are means \pm SE. $n=$ at least 4 for each genotype. ${ }^{*} P<0.05$ versus WT controls; ${ }^{\#} P=0.06$ versus WT controls; ${ }^{+} P=0.05$ versus DCM; ${ }^{\dagger} P<0.05$ versus DCM.

implicated in such regulatory functions are thought to exist in microdomains spatially distinct from bulk SR $\mathrm{Ca}^{2+}$ stores [30], which are probably regulated by sarcolemmal $\mathrm{Ca}^{2+}$ pumps such as the plasma membrane $\mathrm{Ca}^{2+}$-ATPases (PMCA). Impairments in cytosolic bulk $\mathrm{Ca}^{2+}$-handling have the potential to perturb the spatial isolation of these $\mathrm{Ca}^{2+}$ microdomains and can lead to a greater reliance on sarcolemmal $\mathrm{Ca}^{2+}$ pumps for $\mathrm{Ca}^{2+}$-clearance. RT-PCR analysis revealed that mRNA levels for PMCA4 were increased in HCM hearts ( $141 \pm 4 \%$ of WT levels) and remained similarly elevated in HCM/HET hearts (Figure 6(e)). In contrast, mRNA levels for PMCA1, which were not significantly altered in HCM hearts, were reduced in HCM/HET hearts (to $80 \pm$ $6 \%$ of WT levels, Figure 6(f)).

As described above, increased myofibrillar $\mathrm{Ca}^{2+}$-buffering in HCM hearts has the potential to antagonize SR $\mathrm{Ca}^{2+}$ sequestration, with effects on SR/ER Ca ${ }^{2+}$-store levels. However, as we have previously demonstrated [22], the amplitude of stimulated $\mathrm{Ca}^{2+}$-transients is only modestly reduced in $\mathrm{HCM}$ myocytes, which raises the possibility that SR/ER $\mathrm{Ca}^{2+}$ stores may be replenished by alternative mechanisms in these hearts. These can include mediators of store-operated $\mathrm{Ca}^{2+}$ entry (SOCE); indeed, expression of mRNAs for both Orail (to $155 \pm 19 \%$ of WT levels) and Stim1 (to $178 \pm 9 \%$ of WT levels), which encode proteins with well-characterized roles in stress-induced SOCE activity [31, 32], was increased in HCM hearts. Unexpectedly, this increase was reversed to WT levels in HCM/HET hearts (Figures 6(g) and 6(h)).

Increased expression or activity of the protein phosphatases calcineurin, protein phosphatase 1 (PP1), and protein phosphatase 2A (PP2A) is strongly associated with pathological hypertrophy and heart failure [33-36]. Protein levels of the catalytic subunits of calcineurin (CnA), PP1 (PP1-C), and PP2A (PP2A-C), which reflect expression of the respective holoenzymes, were assessed. While $\mathrm{CnA}$ and PP2A-C expression were comparable between HCM and 


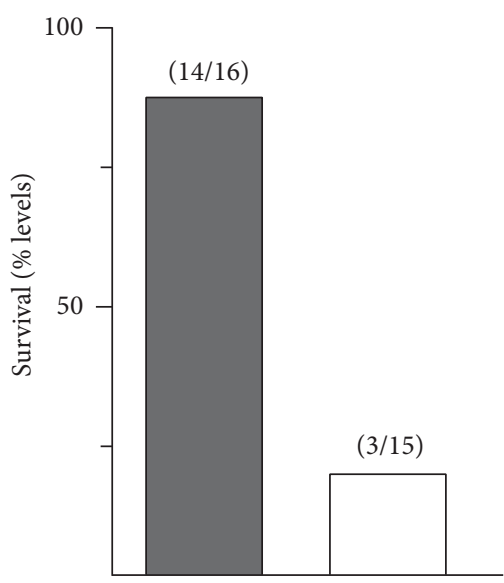

HCM

(a)

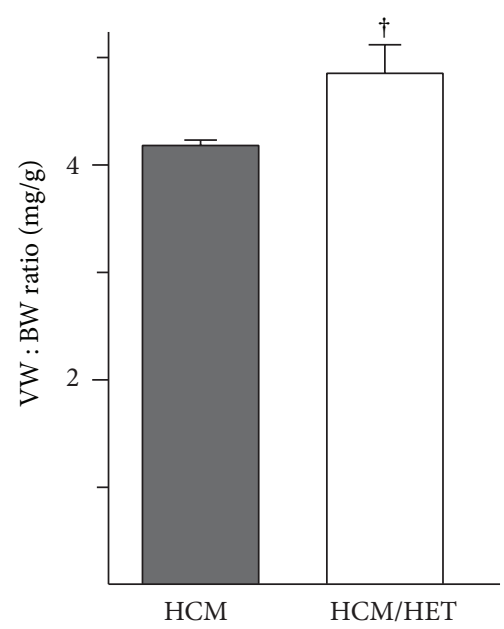

(d)

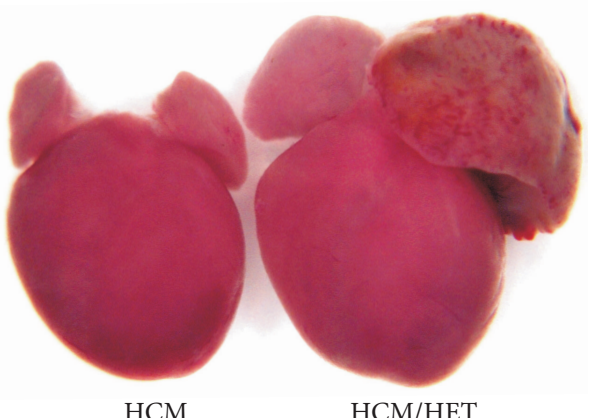

(b)

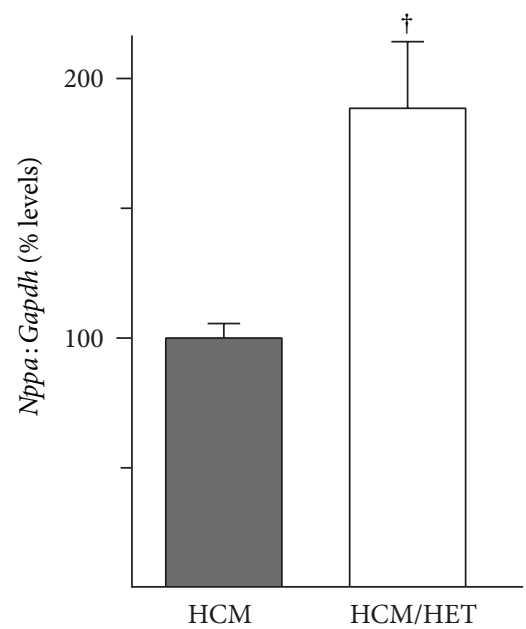

(e)

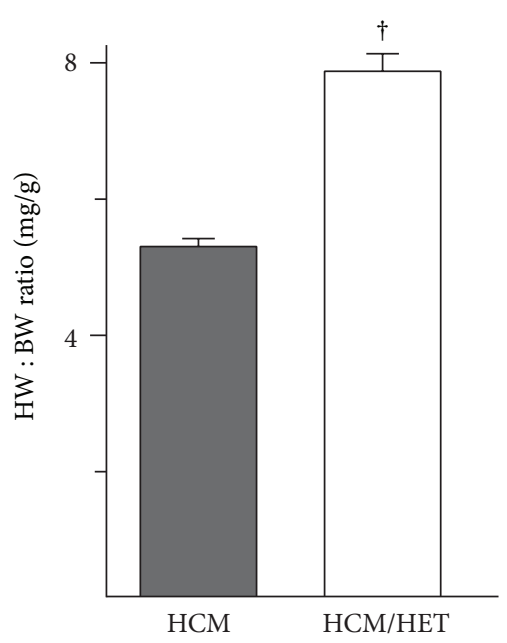

(c)

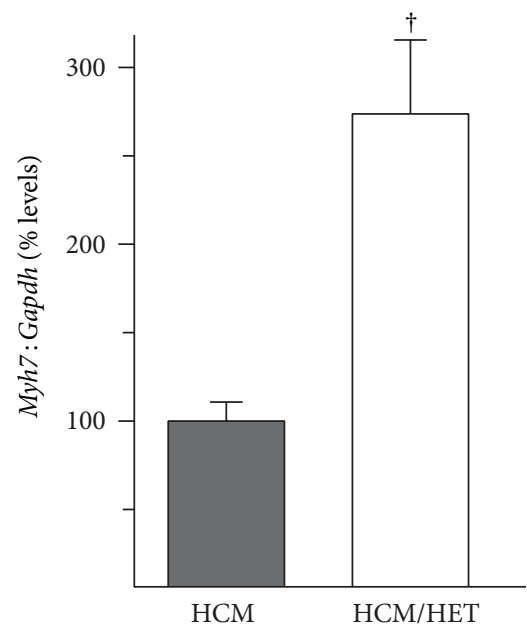

(f)

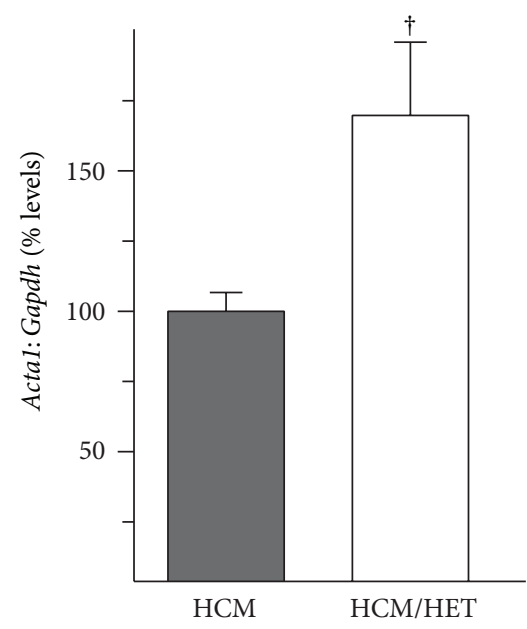

(g)

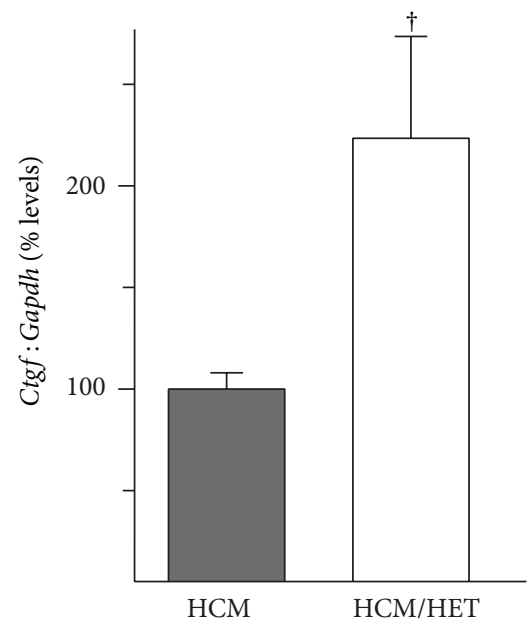

(h)

FIGURE 5: Effects of Atp2a2 heterozygosity in a transgenic model of increased myofibrillar $\mathrm{Ca}^{2+}$ sensitivity. WT mice, mice expressing the Glu180Gly mutant $\alpha$-tropomyosin, which causes hypertrophic cardiomyopathy (HCM), and double mutant HCM/Atp2a2 $2^{+/-}$(HCM/HET) mice were analyzed. Survival of HCM and HCM/HET mice was assessed at 5 weeks of age (a). Gross morphometry at 4 weeks of age showed (b) overt remodeling, (c) increased heart weight: body weight ratios (HW:BW), and (d) increased ventricular weight: body weight ratios (VW : BW) in HCM/HET mice. RT-PCR shows elevated mRNA levels in HCM/HET hearts for (e) atrial natriuretic peptide (Nppa), (f) $\beta$ myosin heavy chain (Myh7), (g) skeletal $\alpha$-actin (Acta1), and (h) connective tissue growth factor (Ctgf). mRNA levels were normalized to Gapdh expression. Values shown are means \pm SE. $n=$ at least 4 for each genotype. ${ }^{\dagger} P<0.05$ versus HCM controls. 


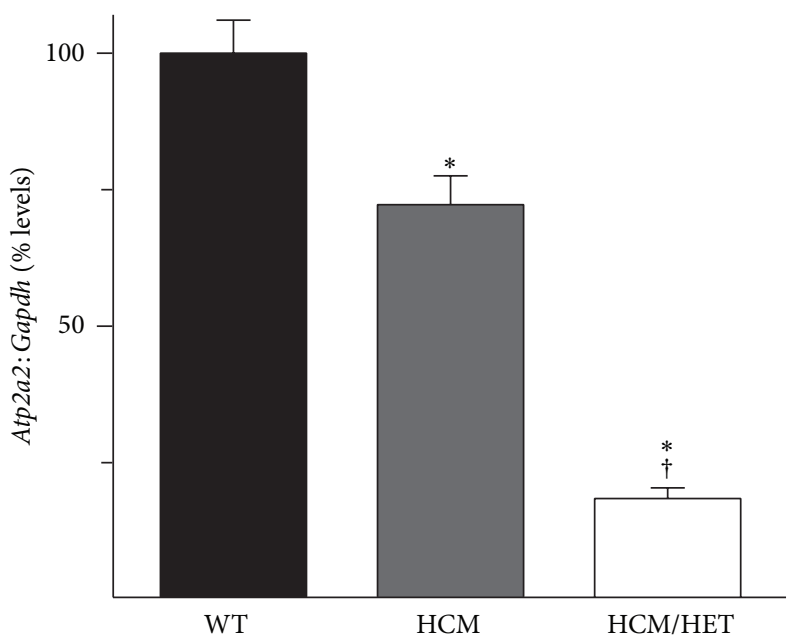

(a)

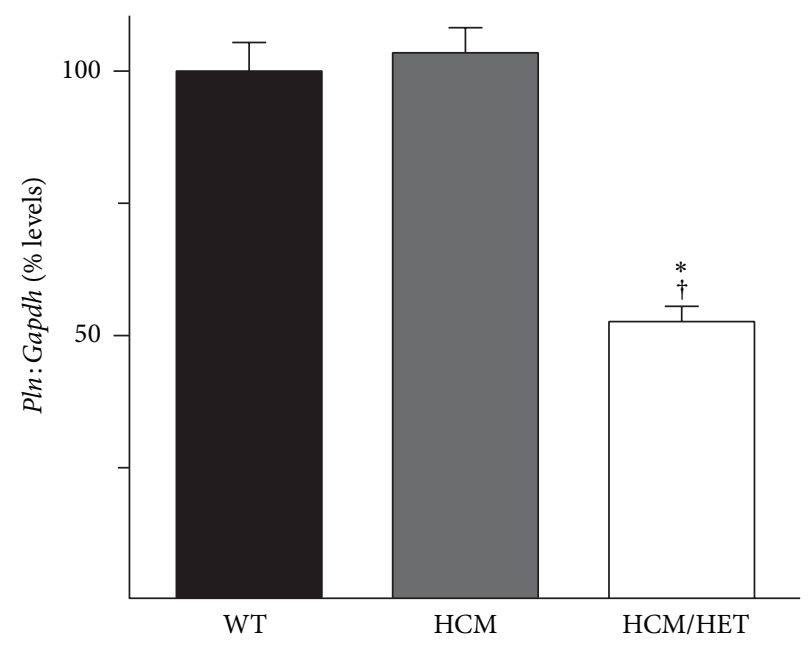

(b)


(c)

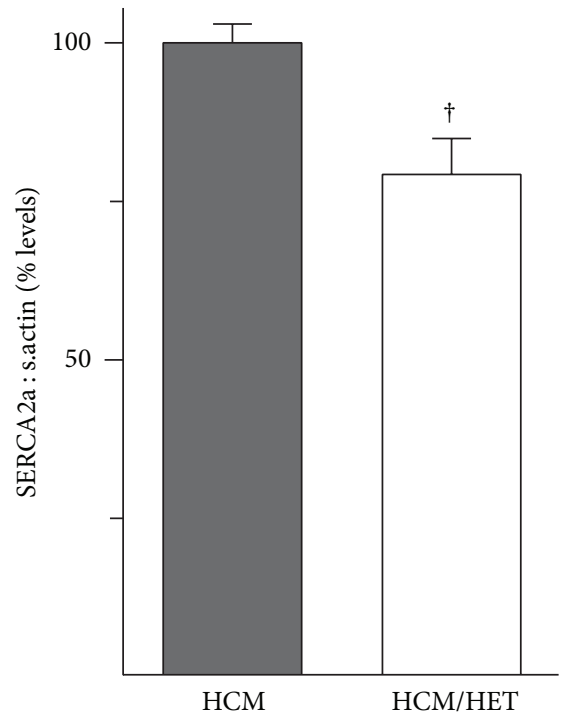

(d)

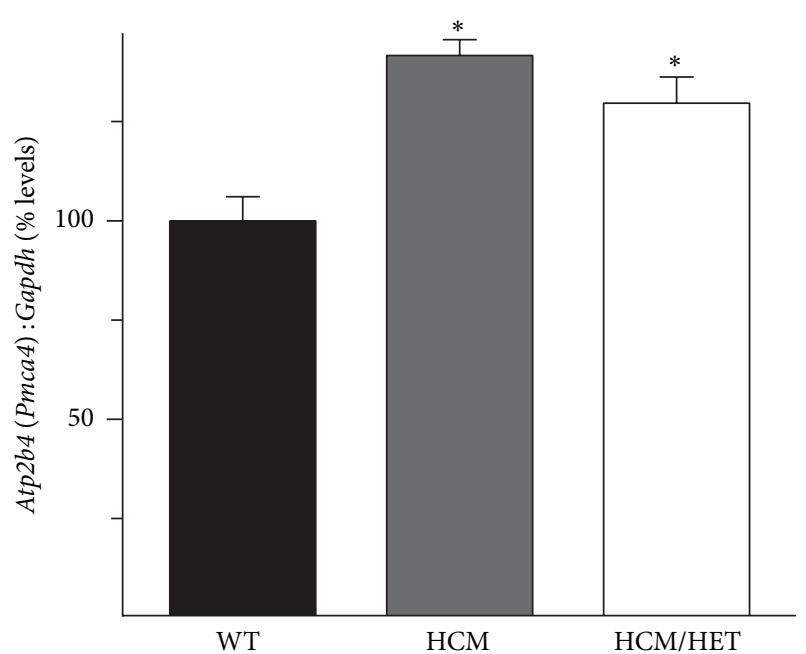

(e)

Figure 6: Continued. 


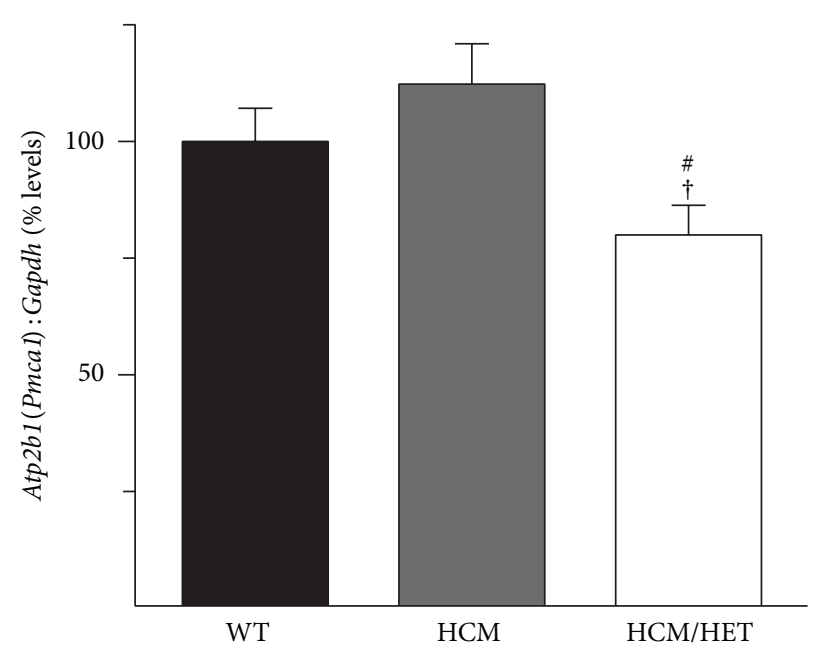

(f)

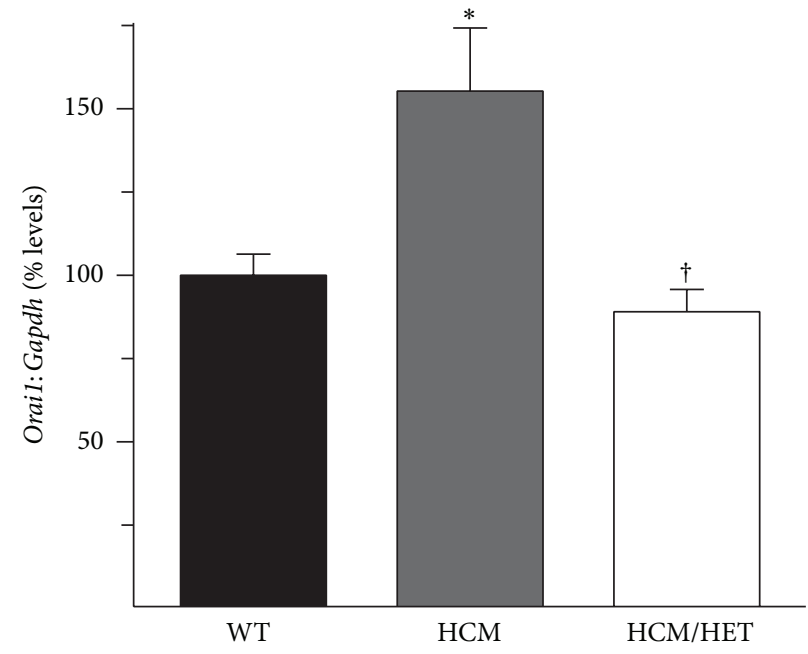

(g)

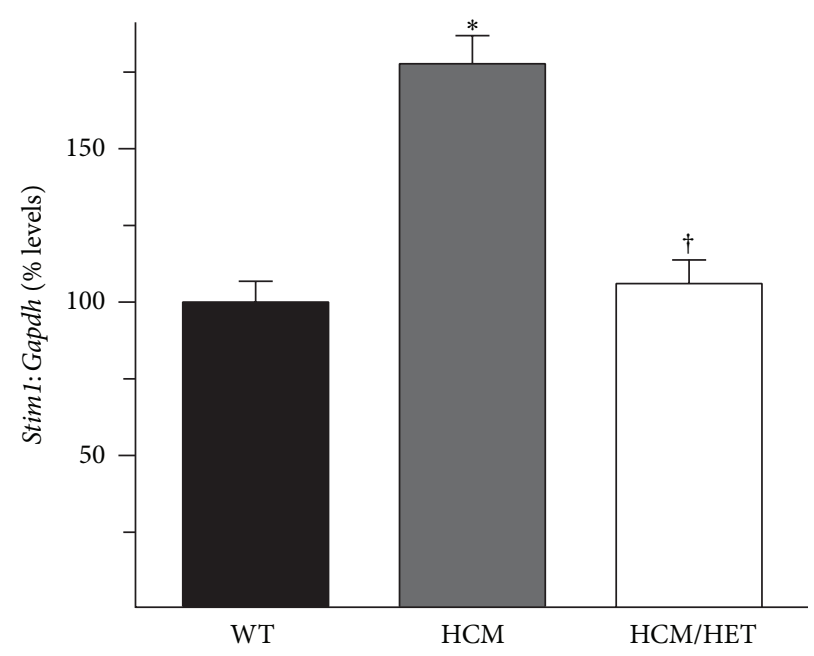

(h)

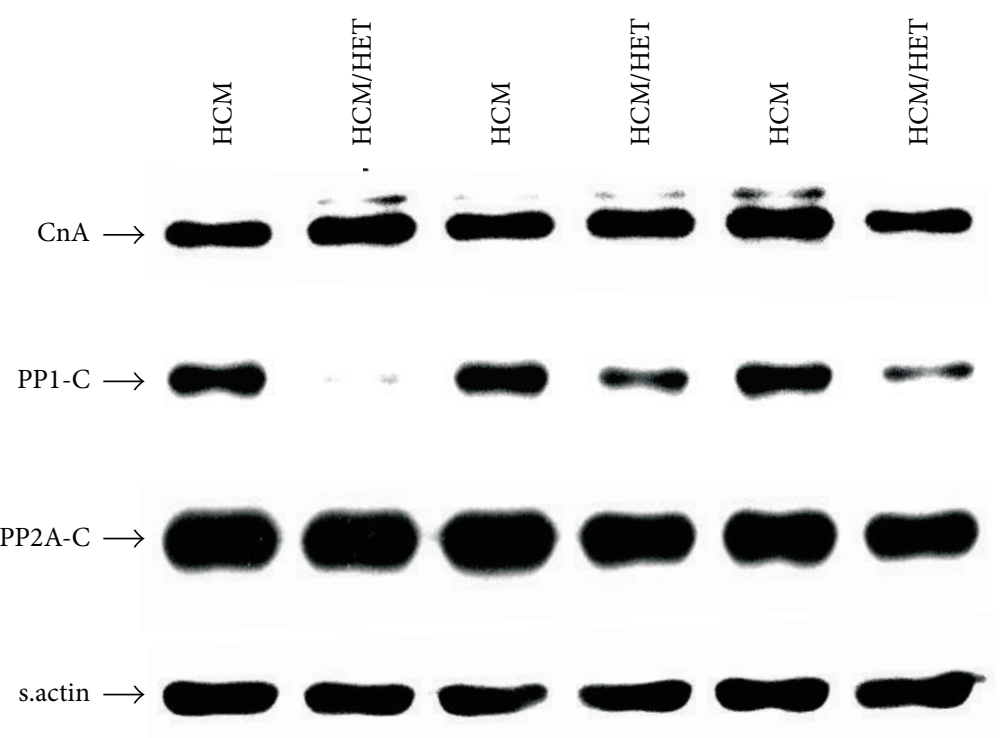

(i)

Figure 6: Continued. 


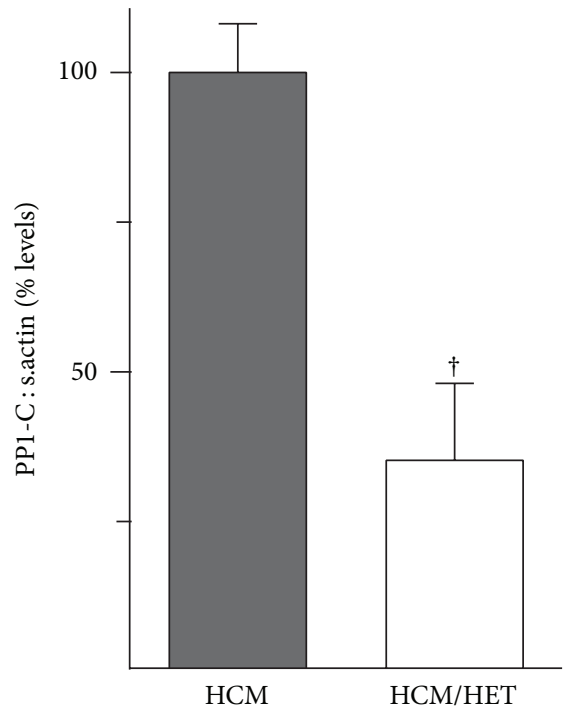

(j)

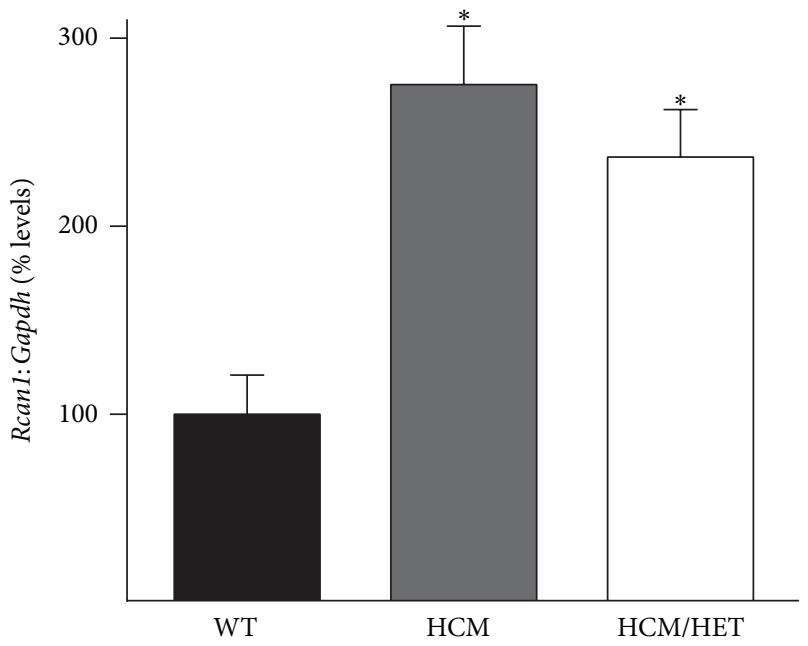

$(\mathrm{k})$

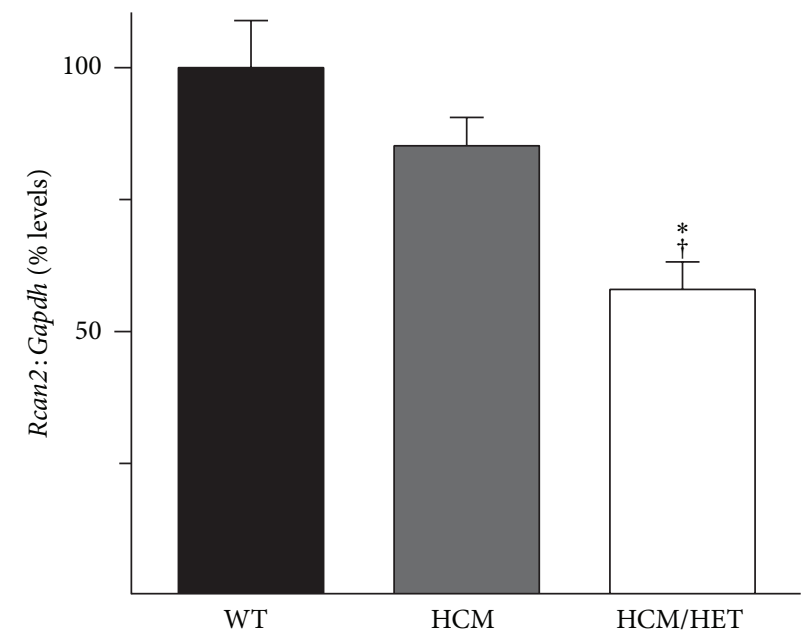

(1)

FIGURE 6: Effects of Atp2a2 heterozygosity on regulators of $\mathrm{Ca}^{2+}$-handling and phosphatases in HCM models. Hearts from WT mice, mice expressing the Glu180Gly mutant $\alpha$-tropomyosin (HCM), and HCM/Atp2a2 $2^{+-}$double mutant (HCM/HET) mice were processed for RTPCR and immunoblots. RT-PCR analysis of mRNA for (a) SERCA2 (Atp2a2) and (b) phospholamban (Pln), (c) immunoblot analysis of SERCA2a and PLN, and (d) quantitation of SERCA2a protein. RT-PCR analyses of mRNA for (e) plasma membrane Ca ${ }^{2+}$-ATPase isoform 4 (Atp2b4), (f) plasma membrane $\mathrm{Ca}^{2+}$-ATPase isoform 1 (Atp2b1), (g) sarcolemmal calcium release-activated calcium modulator 1 (Orail), and (h) stromal interaction molecule 1 (Stim1) and (i) immunoblot analyses of the catalytic subunits of calcineurin (CnA), protein phosphatase 1, (PP1-C), and protein phosphatase 2A (PP2A-C) in HCM and HCM/HET hearts. (j) Quantitation of PP1-C protein levels. RT-PCR analyses of mRNA for (k) regulator of calcineurin 1 (Rcanl) and (l) regulator of calcineurin 2 (Rcan2). mRNA levels were normalized to Gapdh and protein levels were normalized to sarcomeric actin (s.actin). Values are means \pm SE. $n=$ at least 4 for each genotype. ${ }^{*} P<0.05$ versus WT controls; ${ }^{\dagger} P<0.05$ versus $\mathrm{HCM} ;{ }^{\#} P=0.08$ versus WT controls.

HCM/HET hearts (Figure 6(i)), PP1-C levels were reduced in $\mathrm{HCM} / \mathrm{HET}$ hearts (Figures 6(i) and 6(j)). To address the possibility that calcineurin activity was augmented in HCM/HET hearts, mRNA levels for regulator of calcineurin 1 (calcipressin-1, Rcan1), a marker of calcineurin activity, were determined by RT-PCR analysis. While there was no difference in Rcanl levels between HCM and HCM/ HET hearts (Figure 6(k)), mRNA levels of Rcan2, which codes for calcipressin-2, were significantly reduced in $\mathrm{HCM} / \mathrm{HET}$ hearts $(58 \pm 5 \%$ of WT levels; Figure 6(l)).

3.7. Expression of ER Stress Markers and Regulators of Apoptosis in HCM/HET Hearts. The reduction in SERCA2a protein levels, coupled with the downregulation of SOCE-related genes, raised the possibility that ER stress was elevated in HCM/HET hearts. We initially assessed expression of key ER stress markers in HCM hearts. RT-PCR analysis revealed 


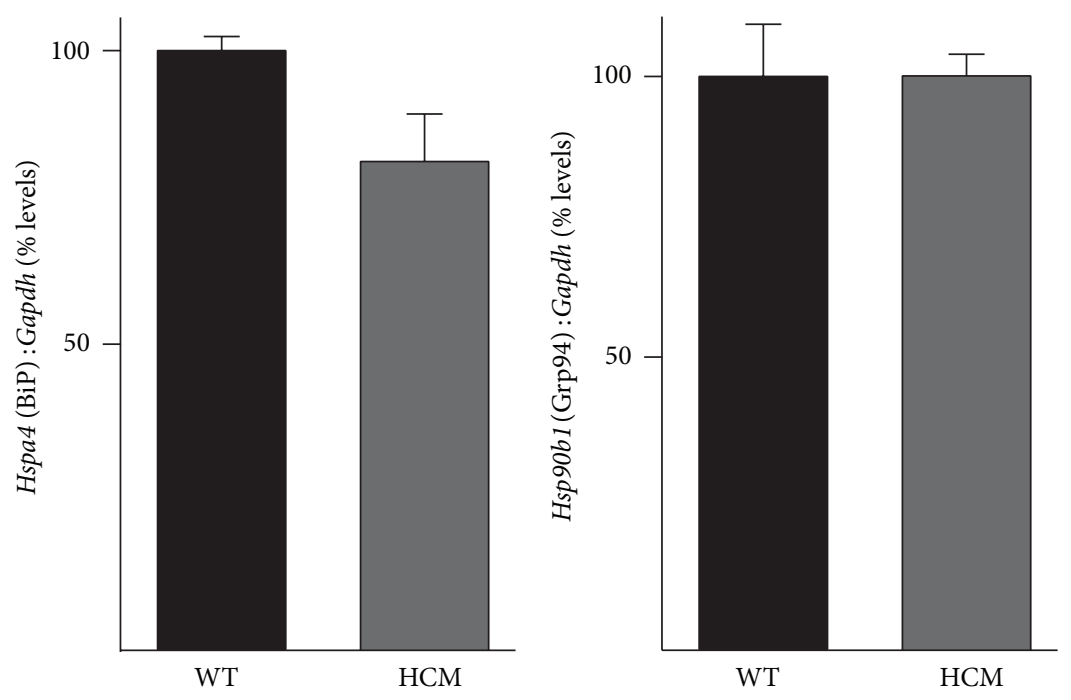

(a)

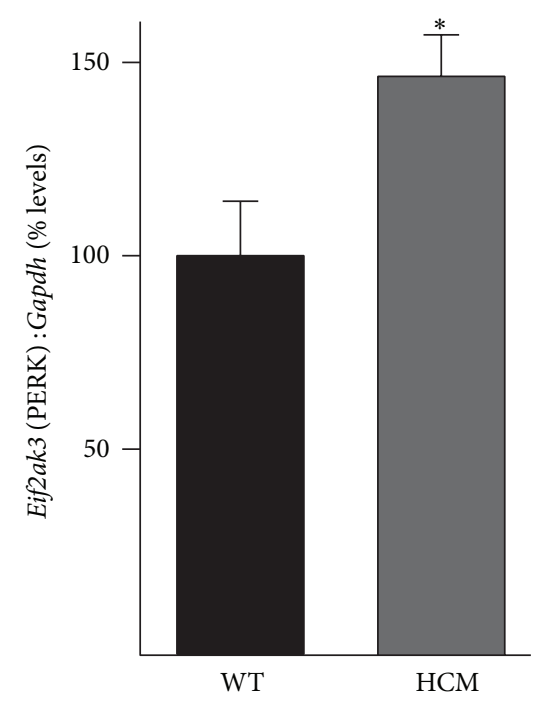

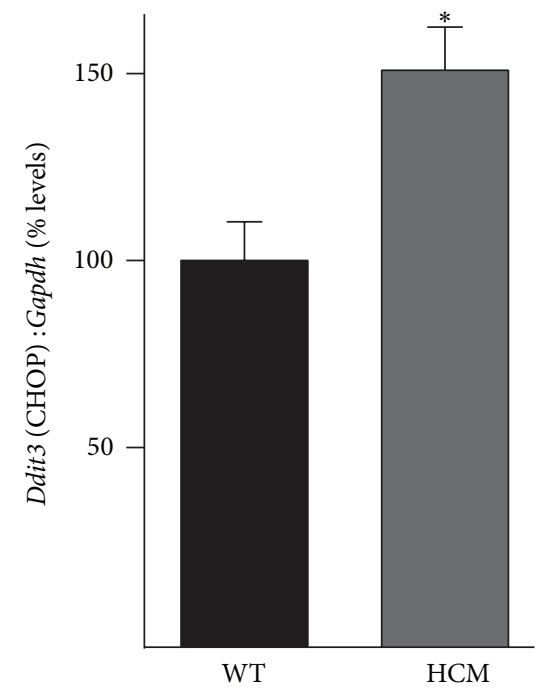

(c)

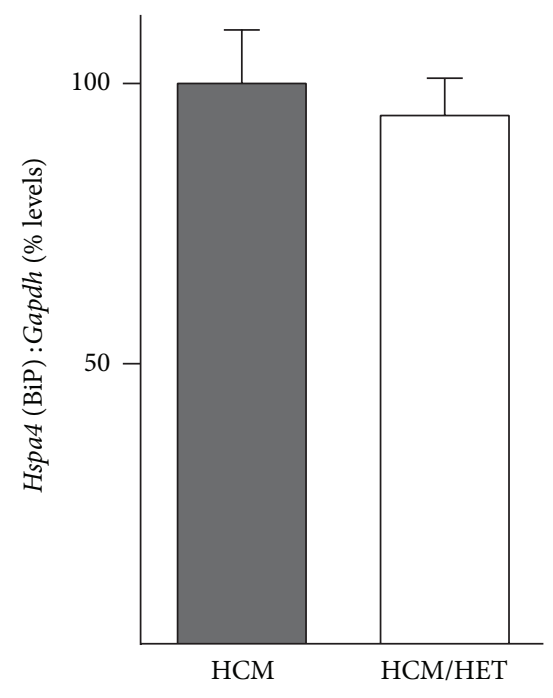

(f)

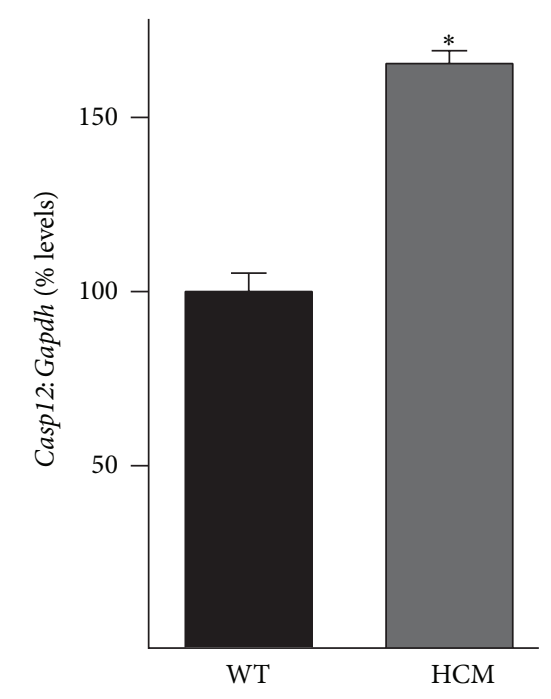

(d)

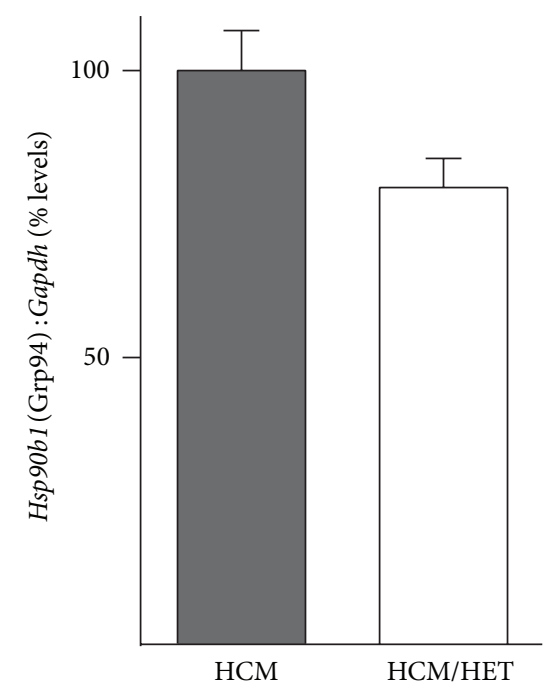

Figure 7: Continued.

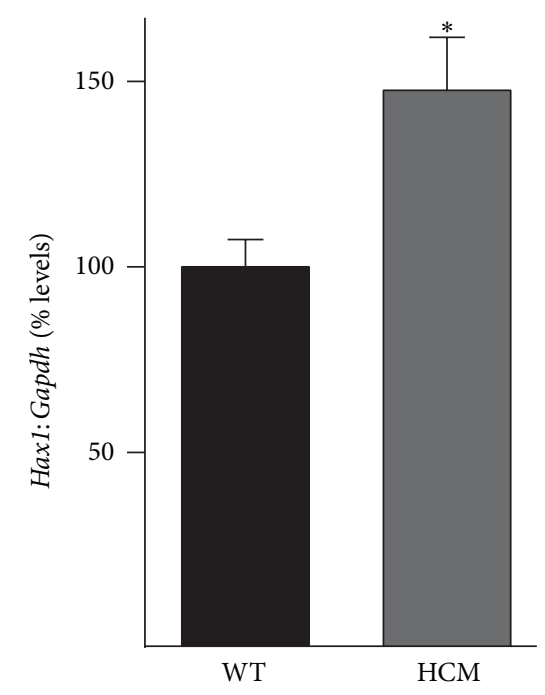

(e)

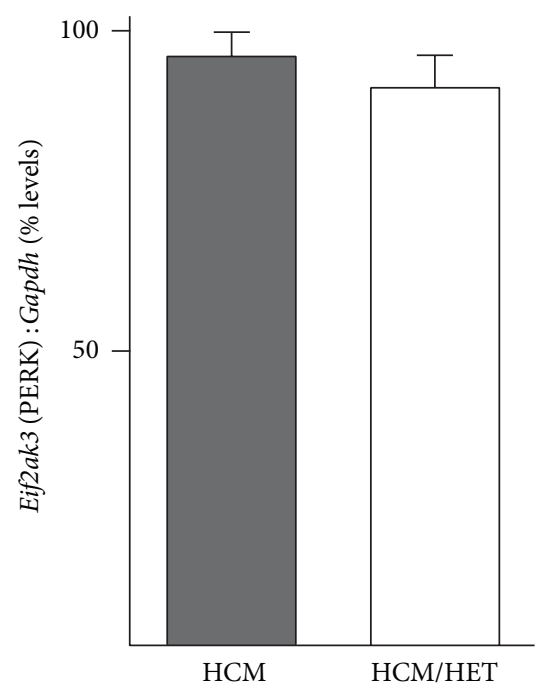

(g) 


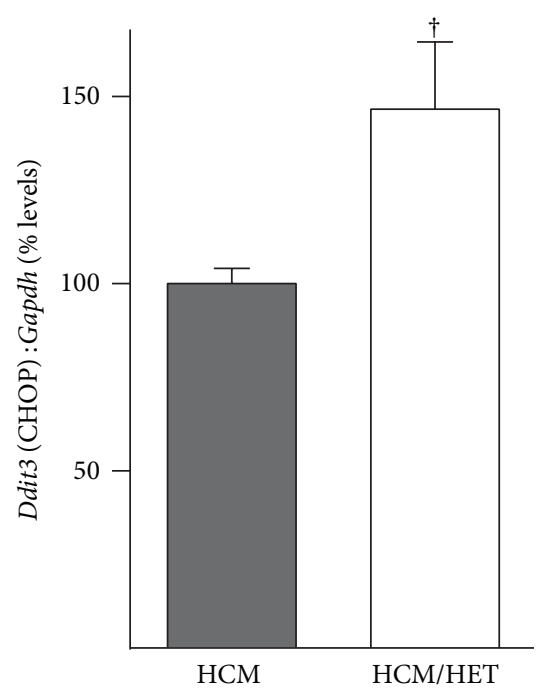

(h)

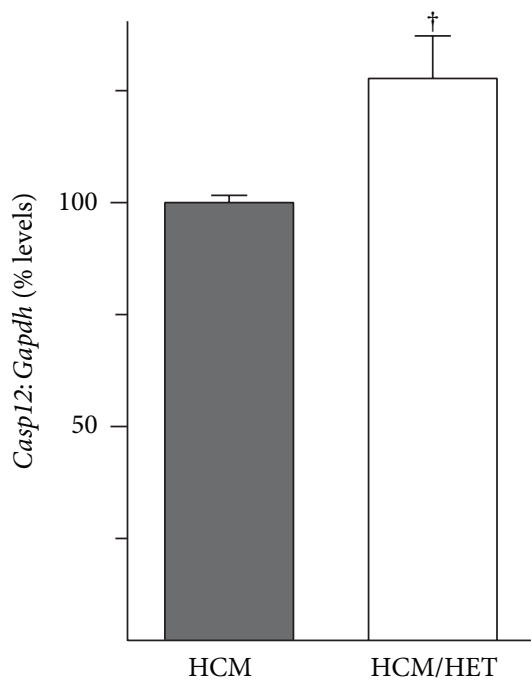

(i)

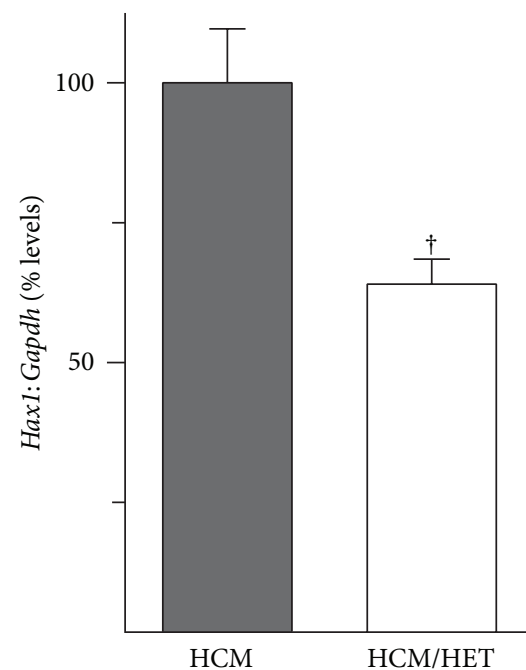

$(\mathrm{j})$

FIGURE 7: Effect of Atp2a2 heterozygosity on markers of ER stress and apoptosis in HCM and HCM/HET hearts. Heart RNA from WT mice, mice expressing the Glu180Gly mutant $\alpha$-tropomyosin (HCM), and HCM/Atp2a2 ${ }^{+/-}$double mutant (HCM/HET) mice was analyzed by RTPCR. Panels (a)-(e) compare WT and HCM; panels (f)-(j) compare HCM and HCM/HET. mRNA levels are shown for (a,f) ER chaperones BiP/GRP78 (Hspa4) and GRP94 (Hsp90b1); (b, g) PERK (Eif2ak3); (c, h) CHOP (Ddit3); (d, i) caspase 12 (Casp12); and (e, j) HCLS1 associated protein X-1 (Haxl) in WT, HCM, and HCM/HET hearts. mRNA levels were normalized to Gapdh. Values shown are means \pm SE. $n=$ at least 4 for each genotype. ${ }^{*} P<0.05$ versus WT controls; ${ }^{\dagger} P<0.05$ versus $\mathrm{HCM}$.

no increase in mRNA for the ER chaperones BiP/GRP78 or GRP94 (Figure 7(a)). However, mRNA for PERK, a major kinase involved in ER stress responses, was increased in HCM hearts (to $147 \pm 12 \%$ of WT levels; Figure 7(b)). Furthermore, mRNAs for the ER stress related proapoptotic proteins $\mathrm{CHOP}$ $(145 \pm 12 \%$ of WT levels; Figure 7(c)) and CASP12 (164 \pm $5 \%$ of WT levels; Figure $7(\mathrm{~d})$ ) were also increased in HCM hearts. These changes were associated with an increase (to $148 \pm 14 \%$ of WT levels; Figure 7(e)) in mRNA for the antiapoptotic protein HCLS1 associated protein X-1 (HAX1), which is localized to mitochondria and the SR [37].

There was no increase in mRNA levels for GRP78, GRP94, or PERK in HCM/HET hearts when compared to HCM controls (Figures $7(\mathrm{f})$ and $7(\mathrm{~g})$ ). However, mRNA levels for CHOP (143 $\pm 16 \%$ of HCM levels; Figure 7(h)) and CASP12 $(128 \pm 9 \%$ of HCM levels; Figure 7(i)) were further elevated in HCM/HET hearts. The increase seen in HAX1 mRNA in HCM hearts was reversed in HCM/HET hearts (to $64 \pm 4 \%$ of HCM levels; Figure 7(j)).

3.8. Effect of Atp2a2 Heterozygosity on Regulators of Energy Metabolism in HCM Hearts. Impaired relaxation resulting from an increase in myofibrillar $\mathrm{Ca}^{2+}$-sensitivity can energetically stress the heart [38]. The resultant dysregulation of energy metabolism has been suggested to contribute to the hypertrophic phenotype associated with sarcomeric mutations that increase myofibrillar $\mathrm{Ca}^{2+}$-sensitivity $[39,40]$. mRNA levels of Ppary, encoding peroxisome proliferatoractivated receptor gamma, a regulator of lipid metabolism that is implicated in the development of pathological hypertrophy [41], were normal in HCM hearts but reduced in HCM/HET hearts (to $81 \pm 6 \%$ of WT levels; Figure $8(\mathrm{a})$ ). Hearts rely predominantly on lipids for their energy supply [42] and FABP3 is the cardiac/muscle-isoform of fatty acid binding proteins, which mediate the intracellular transport of long-chain fatty acids. Expression of the Fabp3 gene was downregulated (to $76 \pm 9 \%$ of WT levels) in HCM hearts and further reduced in HCM/HET hearts (to $49 \pm 5 \%$ of WT levels; Figure 8(b)). Mitochondrial uptake of longchain fatty acids is mediated by carnitine palmitoyltransferase lb (CPT1b), the mRNA levels for which were reduced in HCM (to $88 \pm 3 \%$ of WT levels) and further reduced in $\mathrm{HCM} / \mathrm{HET}$ (to $61 \pm 2 \%$ of WT levels) hearts (Figure 8(c)). CPT1b-mediated transfer of long-chain fatty acids can be inhibited by malonyl CoA, which is generated from acetylCoA by acetyl-CoA carboxylase beta (encoded by Acacb). While Acacb mRNA levels trended lower in HCM hearts, they were downregulated in HCM/HET hearts (to $58 \pm 7 \%$ of WT levels; Figure 8(d)). In addition, mRNA levels for acyl-CoA oxidase 1 (Acoxl), which is the first enzyme of the $\beta$-oxidation pathway, were also reduced (to $64 \pm 5 \%$ of WT levels) in HCM/HET hearts (Figure 8(e)). Besides lipids, hearts also utilize glucose as an energy source [42]. Glucose uptake in heart is mediated by members of $\operatorname{Slc} 2 a$ glucose transporter family, of which GLUT4 (Slc2a4) is the predominant isoform in cardiac myocytes. mRNA levels for GLUT4 were reduced (to $80 \pm 2 \%$ of WT levels) in HCM hearts, with a more pronounced reduction (to $47 \pm 2 \%$ of WT levels) in HCM/HET hearts (Figure 8(f)). 


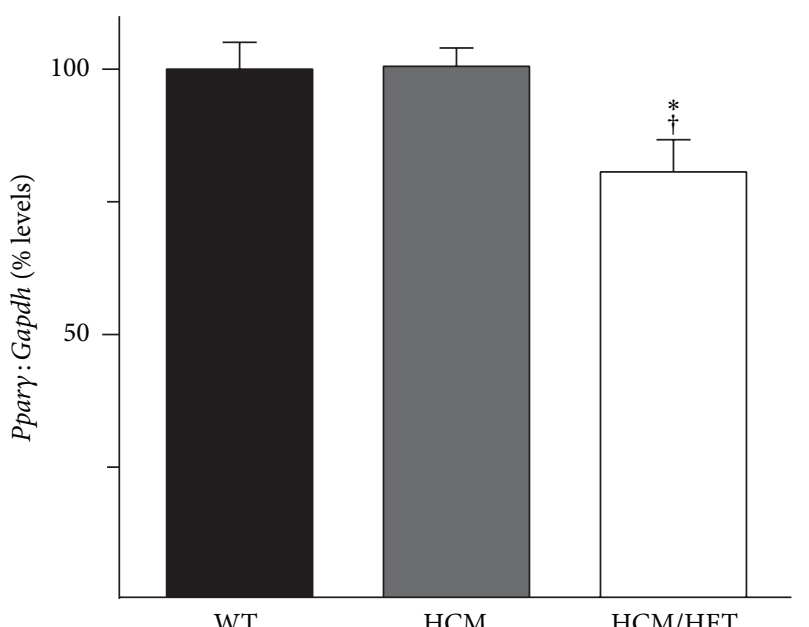

(a)

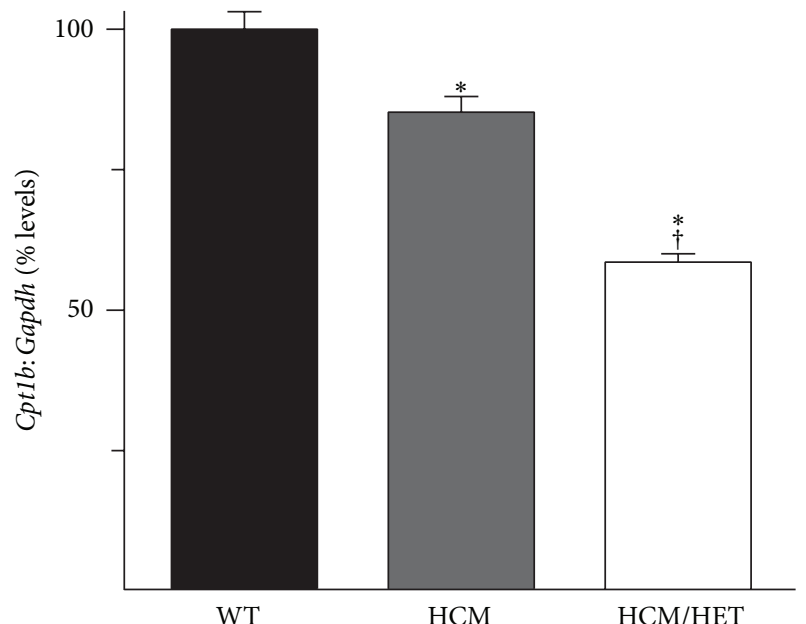

(c)

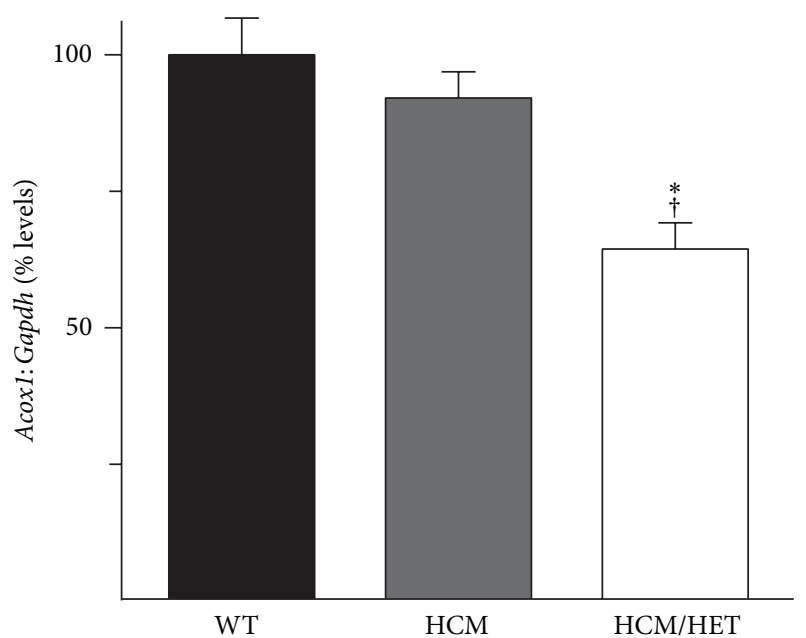

(e)

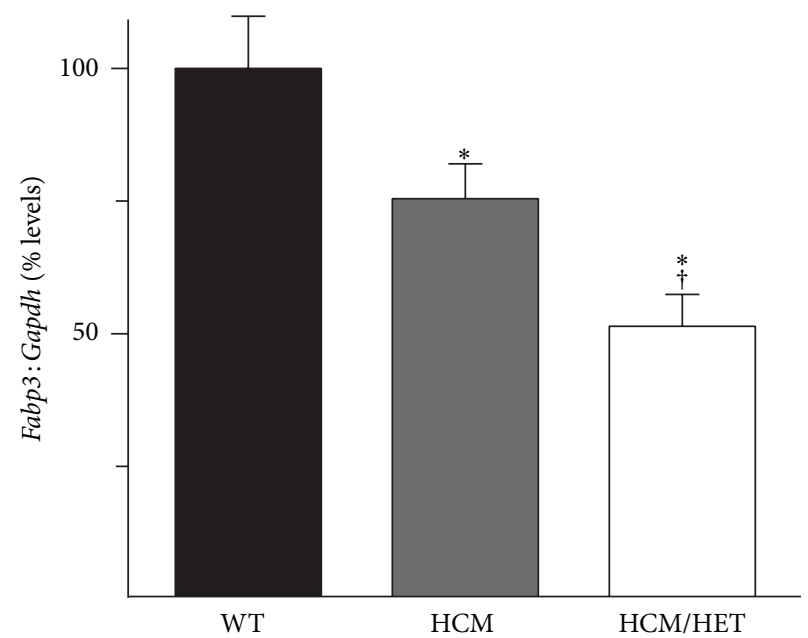

(b)

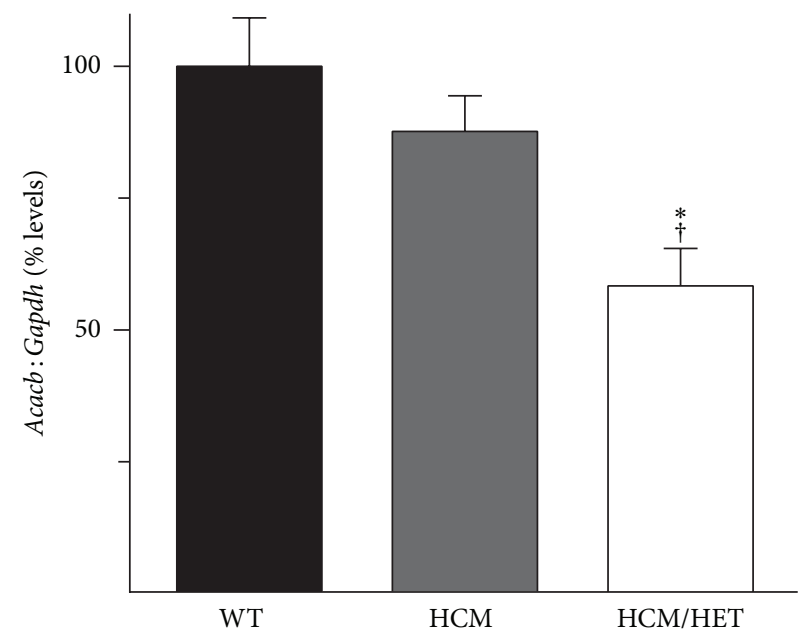

(d)

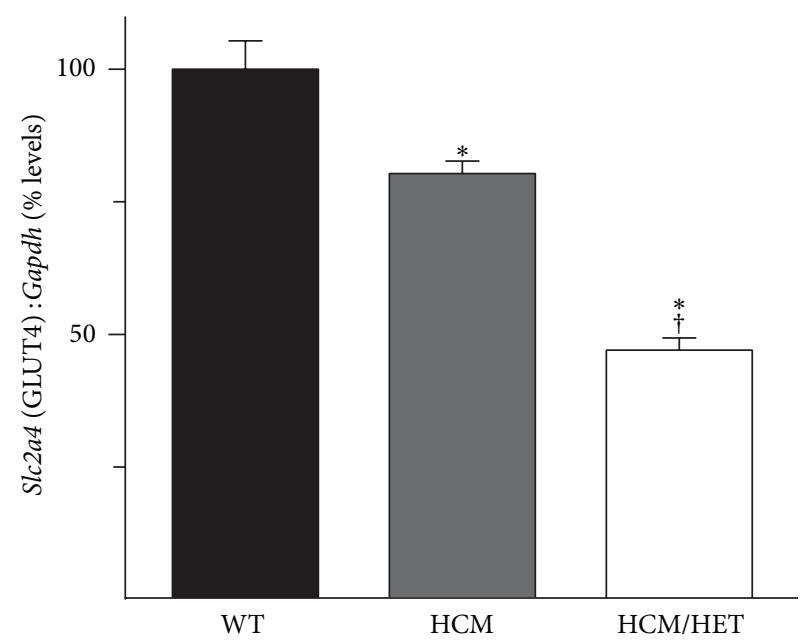

(f)

FIGURE 8: Effect of Atp2a2 heterozygosity on regulators of energy metabolism in HCM and HCM/HET hearts. Heart RNA from WT mice, mice expressing the Glu180Gly mutant $\alpha$-tropomyosin (HCM), and HCM/Atp2a2 ${ }^{+/-}$double mutant (HCM/HET) mice was analyzed by RTPCR. mRNA levels are shown for (a) peroxisome proliferator-activated receptor gamma (Ppar $\gamma$ ); (b) fatty acid binding protein 3 (Fabp3); (c) the muscle-isoform of carnitine palmitoyltransferase 1 (Cpt1b); (d) acetyl CoA-carboxylase beta (Acacb); (e) acyl-CoA oxidase 1 (Acoxl); and (f) the GLUT4 glucose transporter (Slc2a4). mRNA levels were normalized to Gapdh. Values are means \pm SE. $n=$ at least 4 for each genotype. ${ }^{*} P<0.05$ versus WT controls; ${ }^{\dagger} P<0.05$ versus HCM. 
TABLE 1: Effect of Atp2a2 heterozygosity on regulators of apoptosis and energy metabolism in DCM and DCM/HET hearts.

\begin{tabular}{lccc}
\hline Gene & & Relative mRNA levels (normalized to Gapdh) & DCM \\
CHOP (Ddit3) & WT & $103 \pm 3$ & $113 \pm 10$ \\
Caspase 12 (Casp12) & $100 \pm 10$ & $122 \pm 3^{*}$ & $120 \pm 16$ \\
HCLS1 associated protein X-1 (Haxl) & $100 \pm 7$ & $91 \pm 3$ & $106 \pm 12$ \\
Fatty acid binding protein 3 (Fabp3) & $100 \pm 5$ & $70 \pm 4^{*}$ & $75 \pm 7^{*}$ \\
Carnitine palmitoyltransferase 1 (Cpt1b) & $100 \pm 7$ & $77 \pm 3^{*}$ & $70 \pm 8^{*}$ \\
Glucose transporter 4 (Slc2a4) & $100 \pm 2$ & $88 \pm 2$ & $95 \pm 8$ \\
\hline
\end{tabular}

${ }^{9}$ Heart RNA from wild-type (WT) mice, transgenic mice expressing Glu154Lys mutant $\alpha$-tropomyosin (DCM), and DCM/Atp2a2 ${ }^{+/-}$double mutant $(\mathrm{DCM} / \mathrm{HET})$ mice was analyzed by RT-PCR. Values are mean $\pm \mathrm{SE} ; n=$ at least 4 for each genotype; ${ }^{*} P<0.05$ versus WT controls.

3.9. Biochemical Effects of Atp2a2 Heterozygosity Seen in HCM/HET Hearts Are Lacking in DCM/HET Hearts. As described above, Atp2a2 heterozygosity did not exacerbate the cardiac disease phenotype of DCM mice. Given the dramatic effects of SERCA2 haploinsufficiency in HCM/HET hearts at the RNA/protein levels, we analyzed DCM/HET hearts to identify possible underlying similarities. Several mRNAs analyzed (Casp12, Fabp3, and Cpt1b) were altered in DCM hearts relative to WT hearts. However, RT-PCR analysis revealed that Atp2a2 heterozygosity did not impact mRNA levels for Ddit3 (CHOP), Casp12, Haxl, Fabp3, Cpt1b, or Slc2a4 (GLUT4) in DCM/HET hearts when compared with DCM hearts (Table 1). Immunoblot analyses of total ventricular homogenates of DCM and DCM/HET mice showed no change in protein levels of CnA, PP1-C, or PP2A-C (data not shown).

\section{Discussion}

While there is strong evidence that ATP2A2 heterozygosity does not impair cardiovascular performance in humans either at rest or during exercise $[6,7]$, the opportunities to elucidate the molecular mechanisms that allow for cardiac function to be preserved in otherwise healthy DD patients are obviously limited. For example, it remains unknown if loss of one ATP2A2 allele elicits the compensatory upregulation of the second functional allele or whether SERCA2a haploinsufficiency in human heart leads to a greater, and apparently effective, reliance on transsarcolemmal $\mathrm{Ca}^{2+}$-flux. There have also been no studies to determine if DD patients are more susceptible to heart failure and decompensation in the context of secondary pathological insults. The Atp2a2 heterozygous mouse model on the inbred FVB/N background offered a unique opportunity to address these questions, as it resembles human DD patients in displaying a skin/keratinized epithelial phenotype with no overt effects on cardiovascular function.

Expression of SERCA2a protein levels at $\sim 80 \%$ of WT levels in HET hearts demonstrated a remarkable ability to upregulate expression of the single functional Atp2a2 allele. This was unexpected given the evidence from transgenic lines that it is difficult to appreciably overexpress SERCA2 protein in mouse hearts $[43,44]$. Given that Atp2a2 mRNA levels were reduced by $\sim 50 \%$ in HET hearts, it is reasonable to assume that the increase in SERCA2a protein results principally from posttranscriptional adaptations; these could include augmented translation, extended SERCA2 protein half-life, and/or diminished SERCA2 protein degradation. The increase seen in LTCC $\alpha 2$ protein levels is consistent with the idea that even modest reductions in SERCA2a expression might lead to a greater reliance on $\mathrm{Ca}^{2+}$-handling across the sarcolemma. While such a shift would help preserve $\mathrm{Ca}^{2+}$-homeostasis, it is likely to place a greater energetic burden on the heart. SERCA2 is unique in its stoichiometry of transporting $2 \mathrm{Ca}^{2+}$ ions/ATP hydrolyzed. In comparison, transsarcolemmal flux of $\mathrm{Ca}^{2+}$ via LTCC-influx, NCX1-efflux (with a stoichiometry of $3 \mathrm{Na}^{+}: 1 \mathrm{Ca}^{2+}$ ), and $\mathrm{Na}^{+} / \mathrm{K}^{+}$-ATPasemediated $\mathrm{Na}^{+}$removal effectively doubles the energy cost of $\mathrm{Ca}^{2+}$-clearance.

Increased PLN phosphorylation can also serve to compensate for the reduction in SERCA2 levels [23], with evidence of a predominant role for phosphorylation at Ser16, but not Thr17, in $\beta$-adrenergic stimulation of inotropy and lusitropy in mouse hearts [45]. Therefore, it is difficult to ascribe any part of the normal $\beta$-adrenergic response seen in HET hearts to increased PLN phosphorylation, given that PS16 levels were unaltered and only PT17 levels were elevated in stimulated HET hearts. While phosphorylation at Thr17 is mediated by $\mathrm{Ca}^{2+} /$ calmodulin-dependent kinase CaMKII, dephosphorylation is mediated by PP1 [46, 47]. Although immunoblot analysis showed no evidence of a reduction in PP1 expression in HET hearts (data not shown), a clear reduction in PP1 expression was observed in HCM/HET hearts relative to HCM controls, probably providing some compensation for the reduction in SERCA2 protein.

Reduction in SERCA2 expression/activity is closely associated with progression of heart disease and failure. While recent clinical trials provide strong evidence that enhancing SERCA2a expression can alleviate impairment of cardiac function [48-50], it remains unclear whether SERCA2a deficiency can itself precipitate decompensation and heart failure. This is highly relevant to DD patients, particularly in the context of aging or secondary pathological conditions affecting the heart. Hypothyroidism, which affects about $4.6 \%$ of the US population [51], is one such condition known to compromise cardiac function $[13,24]$ and reduce SERCA2 
expression $[20,25]$. The finding that Atp2a2 heterozygosity does not exacerbate the effects of hypothyroidism in mice may result from the switch in myosin heavy chain (MHC) isoforms from $\alpha$-MHC to the slower $\beta$-MHC, which occurs in hypothyroidism, and is known to be energetically favorable [52]. Whether the effects of hypothyroidism are similarly unaffected by SERCA2 haploinsufficiency in human DD patients is an issue that remains to be clarified; it should be evaluated with no preconceptions, as the lack of an effect in the rodent model does not discount possible consequences in human DD patients.

A relative increase in $\beta$-MHC levels, which commonly occurs in diseased conditions, renders hearts more susceptible to chronic myocardial stress [53]. Although this outcome was not tested in the context of hypothyroidism in HET mice, we investigated the effects of Atp2a2 heterozygosity in models of altered myofibrillar $\mathrm{Ca}^{2+}$-sensitivity, which impose significant chronic contractile stress and are closely associated with the pathogenesis of cardiomyopathies and heart failure [54]. DCM mice, with reduced myofibrillar $\mathrm{Ca}^{2+}$-sensitivity, have been reported to develop hypertrophy at 2 months of age, with significant dilation and myocyte disarray occurring by 5 months and mice starting to die at 4-6 months of age [16]. These effects were shown to be associated with downregulation of SERCA2a, consistent with the possibility that Atp2a2 heterozygosity would hasten disease pathogenesis. The absence of such an effect in DCM/HET mice however revealed that SERCA2 haploinsufficiency has limited effects in the context of reduced myofibrillar $\mathrm{Ca}^{2+}$-sensitivity, which occurs during pathological conditions such as inflammation and sepsis [55].

The rapid onset of hypertrophy and decompensation in $\mathrm{HCM} / \mathrm{HET}$ mice on the other hand strongly suggests that disease pathogenesis associated with increased myofibrillar $\mathrm{Ca}^{2+}$-sensitivity may be exacerbated in DD patients. The catastrophic effects of SERCA2 haploinsufficiency in HCM/HET occurred despite SERCA2a protein levels being reduced by just $23 \%$ compared to levels in HCM hearts. This indicates that relatively modest reductions in SERCA2a expression can have profound effects in hearts with increased myofibrillar $\mathrm{Ca}^{2+}$-sensitivity. This effect is likely due to the fact that, besides factors such as mechanical load and sarcolemmal $\mathrm{Ca}^{2+}$-flux, dissociation of $\mathrm{Ca}^{2+}$ from myofibrils is facilitated by SERCA2-mediated $\mathrm{Ca}^{2+}$-clearance [56]. The importance of this function is expected to be amplified in the context of elevated myofibrillar $\mathrm{Ca}^{2+}$-sensitivity, making even small changes in SERCA2 expression/function highly consequential. Recent reports that elevating SERCA2 activity, either by increased expression of SERCA2a or ablation of phospholamban, attenuates disease progression in HCM mice $[57,58]$ are consistent with this hypothesis.

Perturbations in cytosolic $\mathrm{Ca}^{2+}$-clearance are also associated with the generation of ventricular arrhythmias [56], which are strongly implicated in progression to heart failure and sudden death $[59,60]$. Targeted SERCA2a gene therapy has been shown, in multiple models, to reduce ventricular arrhythmias in addition to improving cardiac function [4]. The highly advanced disease condition observed in
HCM/HET mice as young as 4 weeks of age precluded effective functional analyses of these mice. However, we examined left ventricular pressure measurements in HET mice and found no evidence of extrasystolic beats or other arrhythmias under either basal conditions or upon maximal $\beta$-adrenergic stimulation (data not shown).

The more rapid decompensation in HCM/HET hearts was associated with a reversal of the increase in Orail and Stiml levels seen in HCM hearts. The incidence of increased SOCE via ORAI1 and STIM1 in pathological hypertrophy has implicated these proteins in disease pathogenesis [31]. However, recent data from knockout models reveal a more nuanced role for these proteins in heart, with effects on $\mathrm{Ca}^{2+}$ homeostasis and the development of compensatory hypertrophy $[61,62]$. Orail deficiency was found to exacerbate loss of cardiac function and hasten progression to dilation and was associated with increased apoptosis [63]. Indeed, levels of proapoptotic Chop and Casp12 were elevated in HCM/HET hearts and notably expression of antiapoptotic Haxl, which was elevated in HCM hearts, was reduced to WT levels in HCM/HET hearts. There is increasing evidence that the antiapoptotic function of the HAX1 protein is linked to its association with SR $\mathrm{Ca}^{2+}$-handling $[37,64]$, where HAX1 has been shown to localize to the SR in a PLN-dependent manner [65]. HAX1 overexpression, which promotes cell survival, downregulates SERCA2 protein levels, whereas SERCA2 overexpression has been shown to antagonize its protective effects $[66,67]$. Therefore, the reversion of HAX1 expression to WT levels may contribute to the preservation of SERCA2 levels in HCM/HET hearts, with potentially maladaptive effects on myocyte cell survival. Further detailed studies will be necessary to fully elucidate possible interactions between HAX1, which has been localized to mitochondria as well [37], and SERCA2 during HCM pathogenesis. Additional perturbations in myocardial energy metabolism, which are strongly implicated in hypertrophy and heart failure [3841], are also likely to contribute to the rapid decompensation observed in HCM/HET hearts. Reductions in both CPT1b and GLUT4 have been shown to promote pathological hypertrophy and heart failure [68-70]. Conversely, we have recently demonstrated that protection against Tm180-related hypertrophy is associated with preservation of CPT1b and GLUT4 expression at WT levels [23].

In conclusion, our data show that the loss of one copy of the Atp2a2 gene, which causes reduced expression of SERCA2a in heart, appears to be benign under normal conditions and even in some disease states. This finding is consistent with the results of studies in human DD patients, in which the loss of one ATP2a2 allele caused no impairment of cardiac performance $[6,7]$. However, our studies with the mouse model of DD also reveal that loss of a single copy of Atp2a 2 can lead to much more rapid decompensation, heart failure, and death in mice carrying an HCM mutation that increases myofibrillar $\mathrm{Ca}^{2+}$ sensitivity. These results suggest that DD patients may be less tolerant of the changes associated with increased myofibrillar $\mathrm{Ca}^{2+}$-sensitivity in heart. In addition to being relevant to pathological conditions such as end-stage heart failure [15], the implications of this finding 
could extend to the use of therapeutic agents that enhance myofibrillar $\mathrm{Ca}^{2+}$-sensitivity in DD patients. For example, although long-term treatment with the $\mathrm{Ca}^{2+}$-sensitizer levosimendan improves cardiac function in a model with cardiomyocyte-specific ablation of SERCA2, it also significantly increases fibrosis in SERCA2-deficient hearts [71]. While extrapolation of results from rodent models to human disease merits caution, our results suggest that progression of some types of heart disease is likely to be exacerbated by DD mutations. DD patients and their physicians should therefore be aware of the possibility of adverse interactions between SERCA2 haploinsufficiency and certain pathological conditions affecting cardiovascular health.

\section{Conflict of Interests}

The authors declare that there is no conflict of interests regarding the publication of this paper.

\section{Acknowledgments}

This work was supported by National Institutes of Health Grants HL061974 (GES) and HL107957 (YW), an American Heart Association Beginning Grant-in-Aid 11BGIA77220005 (VP), and a Near Horizons Pilot grant from the University of Cincinnati Heart, Lung and Vascular Institute (VP and JNL). The authors thank Maureen Bender for excellent animal husbandry.

\section{References}

[1] A. Sakuntabhai, V. Ruiz-Perez, S. Carter et al., "Mutations in ATP2A2, encoding a $\mathrm{Ca}^{2+}$ pump, cause Darier disease," Nature Genetics, vol. 21, no. 3, pp. 271-277, 1999.

[2] A. Hovnanian, "Darier's disease: from dyskeratosis to endoplasmic reticulum calcium ATPase deficiency," Biochemical and Biophysical Research Communications, vol. 322, no. 4, pp. 12371344, 2004.

[3] M. Periasamy, P. Bhupathy, and G. J. Babu, "Regulation of sarcoplasmic reticulum $\mathrm{Ca}^{2+}$ ATPase pump expression and its relevance to cardiac muscle physiology and pathology," Cardiovascular Research, vol. 77, no. 2, pp. 265-273, 2008.

[4] M. B. Sikkel, C. Hayward, K. T. MacLeod, S. E. Harding, and A. R. Lyon, "SERCA2a gene therapy in heart failure: an antiarrhythmic positive inotrope," British Journal of Pharmacology, vol. 171, no. 1, pp. 38-54, 2014.

[5] Y. Miyauchi, T. Daiho, K. Yamasaki et al., "Comprehensive analysis of expression and function of 51 sarco(endo)plasmic reticulum $\mathrm{Ca}^{2+}$-ATPase mutants associated with darier disease," The Journal of Biological Chemistry, vol. 281, no. 32, pp. 2288222895, 2006.

[6] S. Tavadia, R. C. Tait, T. A. McDonagh, and C. S. Munro, "Platelet and cardiac function in Darier's disease," Clinical and Experimental Dermatology, vol. 26, no. 8, pp. 696-699, 2001.

[7] B. M. Mayosi, A. Kardos, C. H. Davies et al., "Heterozygous disruption of SERCA2a is not associated with impairment of cardiac performance in humans: implications for SERCA2a as a therapeutic target in heart failure," Heart, vol. 92, no. 1, pp. 105109, 2006.
[8] M. Periasamy, T. D. Reed, L. H. Liu et al., "Impaired cardiac performance in heterozygous mice with a null mutation in the sarco(endo)plasmic reticulum $\mathrm{Ca}^{2+}$-ATPase isoform 2 (SERCA2) gene," Journal of Biological Chemistry, vol. 274, no. 4, pp. 2556-2562, 1999.

[9] L. H. Liu, G. P. Boivin, V. Prasad, M. Periasamy, and G. E. Shull, "Squamous cell tumors in mice heterozygous for a null allele of Atp2a2, encoding the sarco(endo)plasmic reticulum $\mathrm{Ca}^{2+}$ ATPase isoform $2 \mathrm{Ca}^{2+}$ pump," Journal of Biological Chemistry, vol. 276, no. 29, pp. 26737-26740, 2001.

[10] V. Prasad, G. P. Boivin, M. L. Miller et al., "Haploinsufficiency of Atp2a2, encoding the sarco(endo)plasmic reticulum $\mathrm{Ca}^{2+}$. ATPase isoform $2 \mathrm{Ca}^{2+}$ pump, predisposes mice to squamous cell tumors via a novel mode of cancer susceptibility," Cancer Research, vol. 65, no. 19, pp. 8655-8661, 2005.

[11] J. E. J. Schultz, B. J. Glascock, S. A. Witt et al., "Accelerated onset of heart failure in mice during pressure overload with chronically decreased SERCA2 calcium pump activity," The American Journal of Physiology-Heart and Circulatory Physiology, vol. 286, no. 3, pp. H1146-H1153, 2004.

[12] S. Huke, L. H. Liu, D. Biniakiewicz, W. T. Abraham, and M. Periasamy, "Altered force-frequency response in non-failing hearts with decreased SERCA pump-level," Cardiovascular Research, vol. 59, no. 3, pp. 668-677, 2003.

[13] J. N. Lorenz and J. Robbins, "Measurement of intraventricular pressure and cardiac performance in the intact closed-chest anesthetized mouse," The American Journal of PhysiologyHeart and Circulatory Physiology, vol. 272, no. 3, pp. H1137H1146, 1997.

[14] D. Fatkin and R. M. Graham, "Molecular mechanisms of inherited cardiomyopathies," Physiological Reviews, vol. 82, no. 4, pp. 945-980, 2002.

[15] J. van der Velden, Z. Papp, R. Zaremba et al., "Increased Ca ${ }^{2+}$ sensitivity of the contractile apparatus in end-stage human heart failure results from altered phosphorylation of contractile proteins," Cardiovascular Research, vol. 57, no. 1, pp. 37-47, 2003.

[16] S. Rajan, R. P. H. Ahmed, G. Jagatheesan et al., "Dilated cardiomyopathy mutant tropomyosin mice develop cardiac dysfunction with significantly decreased fractional shortening and myofilament calcium sensitivity," Circulation Research, vol. 101, no. 2, pp. 205-214, 2007.

[17] T. M. Olson, N. Y. Kishimoto, F. G. Whitby, and V. V. Michels, "Mutations that alter the surface charge of alpha-tropomyosin are associated with dilated cardiomyopathy," Journal of Molecular and Cellular Cardiology, vol. 33, no. 4, pp. 723-732, 2001.

[18] R. Prabhakar, G. P. Boivin, I. L. Grupp et al., "A familial hypertrophic cardiomyopathy $\alpha$-tropomyosin mutation causes severe cardiac hypertrophy and death in mice," Journal of Molecular and Cellular Cardiology, vol. 33, no. 10, pp. 1815-1828, 2001.

[19] L. Thierfelder, H. Watkins, C. MacRae et al., "Alpha-Tropomyosin and cardiac troponin T mutations cause familial hypertrophic cardiomyopathy: A disease of the sarcomere," Cell, vol. 77, no. 5, pp. 701-712, 1994.

[20] T. D. Reed, G. J. Babu, Y. Ji et al., “The expression of SR calcium transport ATpase and the $\mathrm{Na}^{+} / \mathrm{Ca}^{2+}$ exchanger are antithetically regulated during mouse cardiac development and in hypo/hyperthyroidism," Journal of Molecular and Cellular Cardiology, vol. 32, no. 3, pp. 453-464, 2000.

[21] A. N. Wansapura, V. M. Lasko, J. B. Lingrel, and J. N. Lorenz, "Mice expressing ouabain-sensitive $\alpha 1-\mathrm{Na}, \mathrm{K}-\mathrm{ATP}$ ase have increased susceptibility to pressure overload-induced cardiac 
hypertrophy," The American Journal of Physiology-Heart and Circulatory Physiology, vol. 300, no. 1, pp. H347-H355, 2011.

[22] N. J. Al Moamen, V. Prasad, I. Bodi et al., "Loss of the AE3 anion exchanger in a hypertrophic cardiomyopathy model causes rapid decompensation and heart failure," Journal of Molecular and Cellular Cardiology, vol. 50, no. 1, pp. 137-146, 2011.

[23] V. Prasad, J. N. Lorenz, V. M. Lasko et al., "Ablation of plasma membrane $\mathrm{Ca}^{2+}$-ATPase isoform 4 prevents development of hypertrophy in a model of hypertrophic cardiomyopathy," Journal of Molecular and Cellular Cardiology, vol. 77, pp. 53-63, 2014.

[24] D. H. MacLennan and E. G. Kranias, "Phospholamban: a crucial regulator of cardiac contractility," Nature Reviews Molecular Cell Biology, vol. 4, no. 7, pp. 566-577, 2003.

[25] I. Klein and S. Danzi, "Thyroid disease and the heart," Circulation, vol. 116, no. 15, pp. 1725-1735, 2007.

[26] M. Arai, K. Otsu, D. H. MacLennan, N. R. Alpert, and M. Periasamy, "Effect of thyroid hormone on the expression of mRNA encoding sarcoplasmic reticulum proteins," Circulation Research, vol. 69, no. 2, pp. 266-276, 1991.

[27] H. Kögler and J. C. Rüegg, "Cardiac contractility: modulation of myofibrillar calcium sensitivity by $\beta$-adrenergic stimulation," Israel Journal of Medical Sciences, vol. 33, no. 1, pp. 1-7, 1997.

[28] K. Brixius, P. Savvidou-Zaroti, U. Mehlhorn, W. Bloch, E. G. Kranias, and R. H. G. Schwinger, "Increased $\mathrm{Ca}^{2+}$-sensitivity of myofibrillar tension in heart failure and its functional implication," Basic Research in Cardiology, vol. 97, supplement 1, pp. I111-I117, 2002.

[29] M. Endoh, "Cardiac $\mathrm{Ca}^{2+}$ signaling and $\mathrm{Ca}^{2+}$ sensitizers," Circulation Journal, vol. 72, no. 12, pp. 1915-1925, 2008.

[30] S. A. Goonasekera and J. D. Molkentin, "Unraveling the secrets of a double life: contractile versus signaling $\mathrm{Ca}^{2+}$ in a cardiac myocyte," Journal of Molecular and Cellular Cardiology, vol. 52, no. 2, pp. 317-322, 2012.

[31] F. R. Giachini, V. V. Lima, J. L. Hannan, F. S. Carneiro, R. C. Webb, and R. C. Tostes, "STIM1/Orail-mediated store-operated $\mathrm{Ca}^{2+}$ entry: the tip of the iceberg," Brazilian Journal of Medical and Biological Research, vol. 44, no. 11, pp. 1080-1087, 2011.

[32] J. Soboloff, B. S. Rothberg, M. Madesh, and D. L. Gill, "STIM proteins: dynamic calcium signal transducers," Nature Reviews Molecular Cell Biology, vol. 13, no. 9, pp. 549-565, 2012.

[33] J. D. Molkentin, J. R. Lu, C. L. Antos et al., "A calcineurindependent transcriptional pathway for cardiac hypertrophy," Cell, vol. 93, no. 2, pp. 215-228, 1998.

[34] J. Neumann, T. Eschenhagen, L. R. Jones et al., "Increased expression of cardiac phosphatases in patients with end-stage heart failure," Journal of Molecular and Cellular Cardiology, vol. 29, no. 1, pp. 265-272, 1997.

[35] M. Yamada, Y. Ikeda, M. Yano et al., "Inhibition of protein phosphatase 1 by inhibitor-2 gene delivery ameliorates heart failure progression in genetic cardiomyopathy.," The FASEB Journal, vol. 20, no. 8, pp. 1197-1199, 2006.

[36] U. Gergs, P. Boknik, I. Buchwalow et al., "Overexpression of the catalytic subunit of protein phosphatase $2 \mathrm{~A}$ impairs cardiac function," Journal of Biological Chemistry, vol. 279, no. 39, pp. 40827-40834, 2004.

[37] S. V. Yap, E. Vafiadaki, J. Strong, and A. Kontrogianni-Konstantopoulos, "HAX-1: a multifaceted antiapoptotic protein localizing in the mitochondria and the sarcoplasmic reticulum of striated muscle cells," Journal of Molecular and Cellular Cardiology, vol. 48, no. 6, pp. 1266-1279, 2010.
[38] A. Kataoka, C. Hemmer, and P. B. Chase, "Computational simulation of hypertrophic cardiomyopathy mutations in Troponin I: influence of increased myofilament calcium sensitivity on isometric force, ATPase and $\left[\mathrm{Ca}^{2+}\right] i$," Journal of Biomechanics, vol. 40, no. 9, pp. 2044-2052, 2007.

[39] J. G. Crilley, E. A. Boehm, E. Blair et al., "Hypertrophic cardiomyopathy due to sarcomeric gene mutations is characterized by impaired energy metabolism irrespective of the degree of hypertrophy," Journal of the American College of Cardiology, vol. 41, no. 10, pp. 1776-1782, 2003.

[40] C. Ferrantini, A. Belus, N. Piroddi, B. Scellini, C. Tesi, and C. Poggesi, "Mechanical and energetic consequences of HCMcausing mutations," Journal of Cardiovascular Translational Research, vol. 2, no. 4, pp. 441-451, 2009.

[41] B. N. Finck, "The PPAR regulatory system in cardiac physiology and disease," Cardiovascular Research, vol. 73, no. 2, pp. 269277, 2007.

[42] G. D. Lopaschuk, J. R. Ussher, C. D. L. Folmes, J. S. Jaswal, and W. C. Stanley, "Myocardial fatty acid metabolism in health and disease," Physiological Reviews, vol. 90, no. 1, pp. 207-258, 2010.

[43] H. He, F. J. Giordano, R. Hilal-Dandan et al., "Overexpression of the rat sarcoplasmic reticulum $\mathrm{Ca}^{2+}$ ATPase gene in the heart of transgenic mice accelerates calcium transients and cardiac relaxation," The Journal of Clinical Investigation, vol. 100, no. 2, pp. 380-389, 1997.

[44] D. L. Baker, K. Hashimoto, I. L. Grupp et al., “Targeted overexpression of the sarcoplasmic reticulum $\mathrm{Ca}^{2+}$-ATPase increases cardiac contractility in transgenic mouse hearts," Circulation Research, vol. 83, no. 12, pp. 1205-1214, 1998.

[45] G. Chu, J. W. Lester, K. B. Young, W. Luo, J. Zhai, and E. G. Kranias, "A single site (Ser16) phosphorylation in phospholamban is sufficient in mediating its maximal cardiac responses to $\beta$ agonists," Journal of Biological Chemistry, vol. 275, no. 49, pp. 38938-38943, 2000.

[46] C. Mundiña-Weilenmann, L. Vittone, M. Ortale, G. C. de Cingolani, and A. Mattiazzi, "Immunodetection of phosphorylation sites gives new insights into the mechanisms underlying phospholamban phosphorylation in the intact heart," The Journal of Biological Chemistry, vol. 271, no. 52, pp. 33561-33567, 1996.

[47] A. Mattiazzi, C. Mundiña-Weilenmann, C. Guoxiang, L. Vittone, and E. Kranias, "Role of phospholamban phosphorylation on Thr17 in cardiac physiological and pathological conditions," Cardiovascular Research, vol. 68, no. 3, pp. 366-375, 2005.

[48] A. Papolos and W. H. Frishman, "Sarcoendoplasmic reticulum calcium transport ATPase 2a: a potential gene therapy target in heart failure," Cardiology in Review, vol. 21, no. 3, pp. 151-154, 2013.

[49] K. Zsebo, A. Yaroshinsky, J. J. Rudy et al., "Long-term effects of AAV1/SERCA2a gene transfer in patients with severe heart failure: analysis of recurrent cardiovascular events and mortality," Circulation Research, vol. 114, no. 1, pp. 101-108, 2014.

[50] B. Greenberg, A. Yaroshinsky, K. M. Zsebo et al., "Design of a phase $2 b$ trial of intracoronary administration of AAV1/ SERCA2a in patients with advanced heart failure: the CUPID 2 trial (calcium up-regulation by percutaneous administration of gene therapy in cardiac disease phase 2b)," Journal of the American College of Cardiology: Heart Failure, vol. 2, no. 1, pp. 84-92, 2014.

[51] S. H. Golden, K. A. Robinson, I. Saldanha, B. Anton, and P. W. Ladenson, "Prevalence and incidence of endocrine and metabolic disorders in the united states: a comprehensive 
review," Journal of Clinical Endocrinology \& Metabolism, vol. 94, no. 6, pp. 1853-1878, 2009.

[52] C. Holubarsch, R. P. Goulette, R. Z. Litten, B. J. Martin, L. A. Mulieri, and N. R. Alpert, "The economy of isometric force development, myosin isoenzyme pattern and myofibrillar ATPase activity in normal and hypothyroid rat myocardium," Circulation Research, vol. 56, no. 1, pp. 78-86, 1985.

[53] M. Krenz and J. Robbins, "Impact of beta-myosin heavy chain expression on cardiac function during stress," Journal of the American College of Cardiology, vol. 44, no. 12, pp. 2390-2397, 2004.

[54] K. A. Palmiter and R. J. Solaro, "Molecular mechanisms regulating the myofilament response to $\mathrm{Ca}^{2+}$ : implications of mutations causal for familial hypertrophic cardiomyopathy," Basic Research in Cardiology, Supplement, vol. 92, supplement 1, pp. 63-74, 1997.

[55] L.-L. Wu, C. Tang, and M.-S. Liu, "Altered phosphorylation and calcium sensitivity of cardiac myofibrillar proteins during sepsis," The American Journal of Physiology-Regulatory Integrative and Comparative Physiology, vol. 281, no. 2, pp. R408-R416, 2001.

[56] H. E. D. J. Ter Keurs and P. A. Boyden, "Calcium and arrhythmogenesis," Physiological Reviews, vol. 87, no. 2, pp. 457-506, 2007.

[57] J. R. Peña, A. C. Szkudlarek, C. M. Warren et al., "Neonatal gene transfer of Serca2a delays onset of hypertrophic remodeling and improves function in familial hypertrophic cardiomyopathy," Journal of Molecular and Cellular Cardiology, vol. 49, no. 6, pp. 993-1002, 2010.

[58] R. D. Gaffin, J. R. Peña, M. S. L. Alves et al., "Long-term rescue of a familial hypertrophic cardiomyopathy caused by a mutation in the thin filament protein, tropomyosin, via modulation of a calcium cycling protein," Journal of Molecular and Cellular Cardiology, vol. 51, no. 5, pp. 812-820, 2011.

[59] J. M. McLenachan, E. Henderson, K. I. Morris, and H. J. Dargie, "Ventricular arrhythmias in patients with hypertensive left ventricular hypertrophy," The New England Journal of Medicine, vol. 317, no. 13, pp. 787-792, 1987.

[60] A. Curcio, D. Torella, C. Iaconetti et al., "MicroRNA-1 downregulation increases connexin 43 displacement and induces ventricular tachyarrhythmias in rodent hypertrophic hearts," PLoS ONE, vol. 8, no. 7, Article ID e70158, 2013.

[61] H. E. Collins, X. Zhu-Mauldin, R. B. Marchase, and J. C. Chatham, "STIM1/Orail-mediated SOCE: current perspectives and potential roles in cardiac function and pathology," The American Journal of Physiology-Heart and Circulatory Physiology, vol. 305, no. 4, pp. H446-H458, 2013.

[62] H. E. Collins, L. He, L. Zou et al., "Stromal interaction molecule 1 is essential for normal cardiac homeostasis through modulation of ER and mitochondrial function," The American Journal of Physiology-Heart and Circulatory Physiology, vol. 306, no. 8, pp. H1231-H1239, 2014.

[63] J. S. Horton, C. L. Buckley, E. M. Alvarez, A. Schorlemmer, and A. J. Stokes, "The calcium release-activated calcium channel Orail represents a crucial component in hypertrophic compensation and the development of dilated cardiomyopathy," Channels, vol. 8, no. 1, pp. 35-48, 2014.

[64] E. Vafiadaki, D. A. Arvanitis, S. N. Pagakis et al., "The antiapoptotic protein HAX-1 interacts with SERCA2 and regulates Its protein levels to promote cell survival," Molecular Biology of the Cell, vol. 20, no. 1, pp. 306-318, 2009.
[65] E. Vafiadaki, D. Sanoudou, D. A. Arvanitis, D. H. Catino, E. G. Kranias, and A. Kontrogianni-Konstantopoulos, "Phospholamban interacts with HAX-1, a mitochondrial protein with antiapoptotic function," Journal of Molecular Biology, vol. 367, no. 1, pp. 65-79, 2007.

[66] E. Vafiadaki, D. A. Arvanitis, S. N. Pagakis et al., "The antiapoptotic protein HAX-1 interacts with SERCA2 and regulates its protein levels to promote cell survival," Molecular Biology of the Cell, vol. 20, no. 1, pp. 306-318, 2009.

[67] W. Zhao, J. R. Waggoner, Z.-G. Zhang et al., "The anti-apoptotic protein HAX-1 is a regulator of cardiac function," Proceedings of the National Academy of Sciences of the United States of America, vol. 106, no. 49, pp. 20776-20781, 2009.

[68] L. He, T. Kim, Q. Long et al., "Carnitine palmitoyltransferase$1 \mathrm{~b}$ deficiency aggravates pressure overload-induced cardiac hypertrophy caused by lipotoxicity," Circulation, vol. 126, no. 14, pp. 1705-1716, 2012.

[69] K. R. Haynie, B. Vandanmagsar, S. E. Wicks, J. Zhang, and R. L. Mynatt, "Inhibition of carnitine palymitoyltransferaselb induces cardiac hypertrophy and mortality in mice," Diabetes, Obesity \& Metabolism, vol. 16, no. 8, pp. 757-760, 2014.

[70] A. A. Domenighetti, V. R. Danes, C. L. Curl, J. M. Favaloro, J. Proietto, and L. M. D. Delbridge, "Targeted GLUT-4 deficiency in the heart induces cardiomyocyte hypertrophy and impaired contractility linked with $\mathrm{Ca}^{2+}$ and proton flux dysregulation," Journal of Molecular and Cellular Cardiology, vol. 48, no. 4, pp. 663-672, 2010.

[71] V. Hillestad, F. Kramer, S. Golz, A. Knorr, K. B. Andersson, and G. Christensen, "Long-term levosimendan treatment improves systolic function and myocardial relaxation in mice with cardiomyocyte-specific disruption of the Serca2 gene," Journal of Applied Physiology, vol. 115, no. 10, pp. 1572-1580, 2013. 


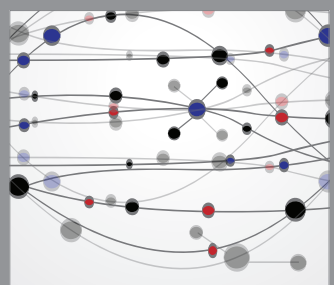

The Scientific World Journal
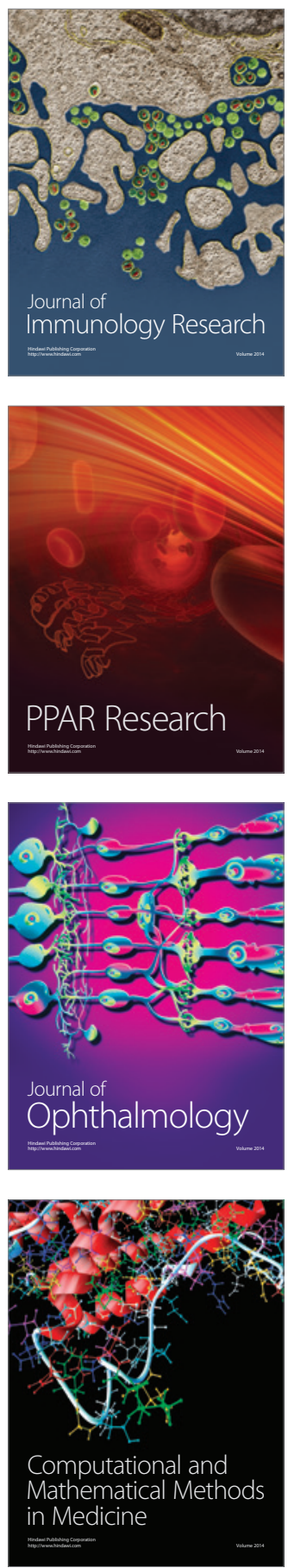

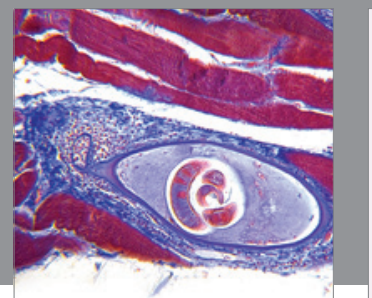

Gastroenterology

Research and Practice
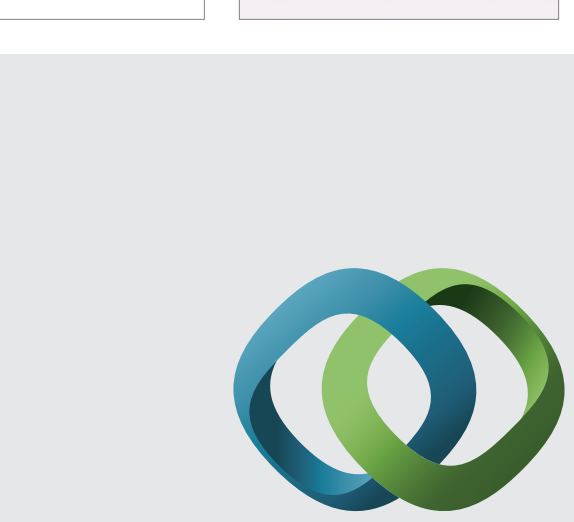

\section{Hindawi}

Submit your manuscripts at

http://www.hindawi.com
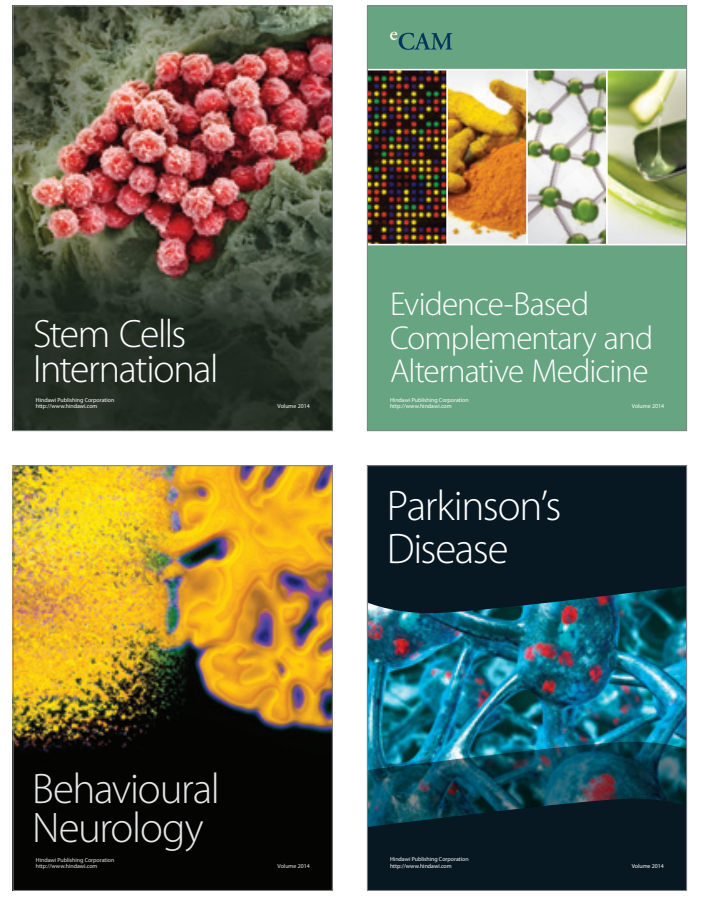
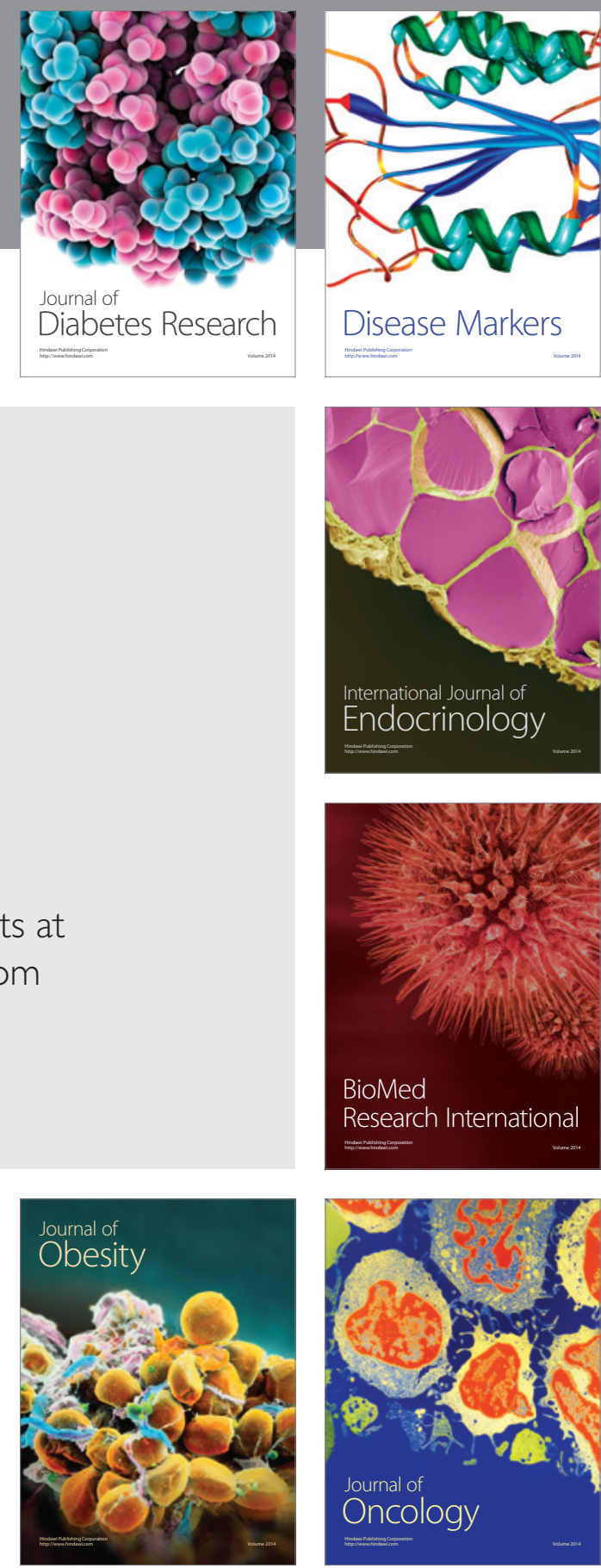

Disease Markers
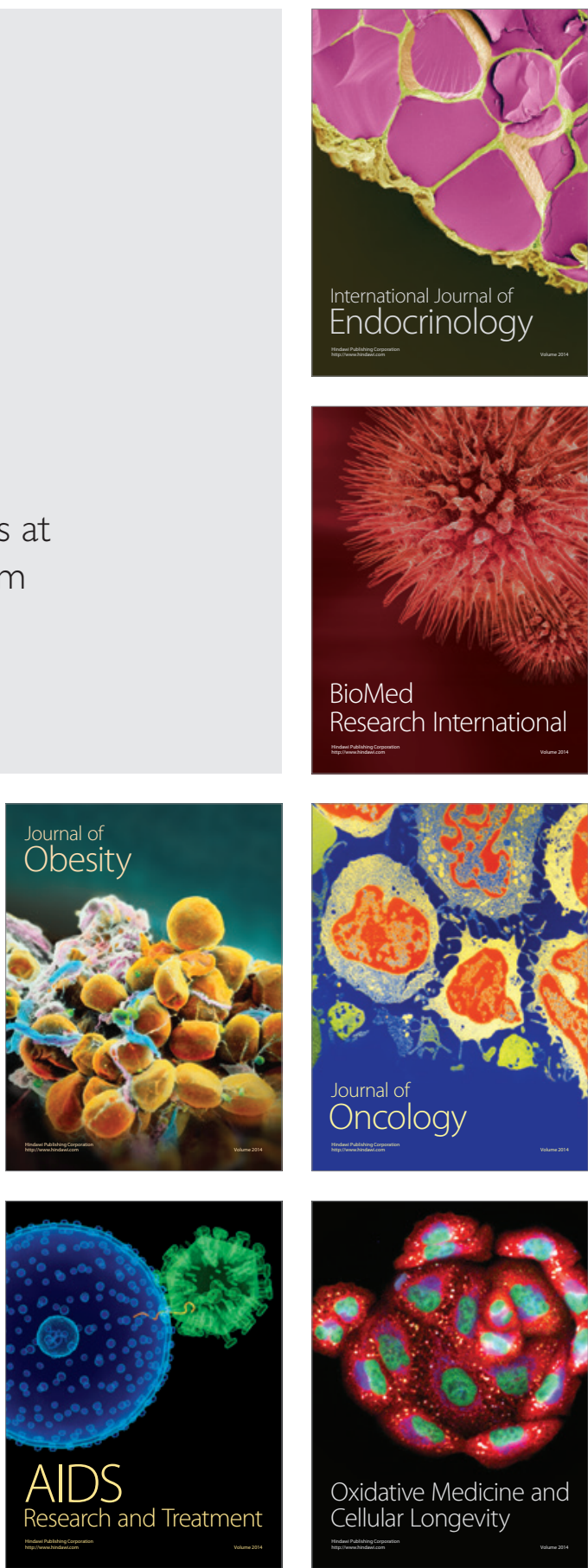